OPEN ACCESS

Edited by:

Christian Sohlenkamp,

Universidad Nacional Autónoma de México, Mexico

Reviewed by:

Eric Déziel,

Institut National de la Recherche

Scientifique (INRS), Canada

Edgardo Sepulveda,

Max Planck Institute

for Developmental Biology, Germany

*Correspondence:

Hai-Hong Wang

wanghh36@scau.edu.cn

tThese authors have contributed equally to this work.

Specialty section:

This article was submitted to Microbial Physiology and Metabolism, a section of the journal

Frontiers in Microbiology

Received: 05 September 2017

Accepted: 25 October 2017

Published: 10 November 2017

Citation:

Ma J-C, Wu Y-Q, Cao D, Zhang W-B and Wang $\mathrm{H}-\mathrm{H}(2017)$ Only Acyl Carrier Protein 1 (AcpP1)

Functions in Pseudomonas aeruginosa Fatty Acid Synthesis.

Front. Microbiol. 8:2186. doi: 10.3389/fmicb.2017.02186

\section{Only Acyl Carrier Protein 1 (AcpP1) Functions in Pseudomonas aeruginosa Fatty Acid Synthesis}

\author{
Jin-Cheng $\mathrm{Ma}^{1 \dagger}$, Yun-Qi $\mathrm{Wu}^{2 \dagger}$, Dan $\mathrm{Cao}^{3+}$, Wen-Bin Zhang ${ }^{1}$ and Hai-Hong Wang ${ }^{1 *}$ \\ ${ }^{1}$ Guangdong Provincial Key Laboratory of Protein Function and Regulation in Agricultural Organisms, College of Life \\ Sciences, South China Agricultural University, Guangzhou, China, ${ }^{2}$ Department of Chemistry and Biomolecular Sciences, \\ Macquarie University, North Ryde, NSW, Australia, ${ }^{3}$ Forensic Science Center of Qingyuan, Qingyuan Public Security \\ Department, Qingyuan, China
}

The genome of Pseudomonas aeruginosa contains three open reading frames, PA2966, PA1869, and PA3334, which encode putative acyl carrier proteins, AcpP1, AcpP2, and AcpP3, respectively. In this study, we found that, although these apo-ACPs were successfully phosphopantetheinylated by $P$. aeruginosa phosphopantetheinyl transferase (PcpS) and all holo-forms of these proteins could be acylated by Vibrio harveyi acyl-ACP synthetase (AasS), only AcpP1 could be used as a substrate for the synthesis of fatty acids, catalyzed by $P$. aeruginosa cell free extracts in vitro, and only acpP1 gene could restore growth in the Escherichia coli acpP mutant strain CY1877. And P. aeruginosa acpP1 could not be deleted, while disruption of acpP2 or $a c p P 3$ in the $P$. aeruginosa genome allowed mutant strains to grow as well as the wild type strain. These findings confirmed that only $P$. aeruginosa AcpP1 functions in fatty acid biosynthesis, and that acpP2 and acpP3 do not play roles in the fatty acid synthetic pathway. Moreover, disruption of acpP2 and acpP3 did not affect the ability of $P$. aeruginosa to produce $N$-acylhomoserine lactones $(\mathrm{AHL})$, but replacement of $P$. aeruginosa acpP1 with $E$. coli acpP caused $P$. aeruginosa to reduce the production of AHL molecules, which indicated that neither $P$. aeruginosa AcpP2 nor AcpP3 can act as a substrate for synthesis of $\mathrm{AHL}$ molecules in vivo. Furthermore, replacement of acpP1 with $E$. coli acpP reduced the ability of $P$. aeruginosa to produce some exo-products and abolished swarming motility in $P$. aeruginosa.

Keywords: Pseudomonas aeruginosa, fatty acid biosynthesis, acyl carrier protein

\section{INTRODUCTION}

Members of the superfamily of $4^{\prime}$-phosphopantetheine (Ppant)-dependent carrier proteins play central roles in fatty acid synthesis, polyketide synthesis and non-ribosomal peptide synthesis (Lai et al., 2006; Smith, 2006; Byers and Gong, 2007; Chan et al., 2009). This family includes the acyl carrier proteins (ACP), aryl carrier proteins and peptidyl carrier proteins, which can exist as a domain within large multifunctional fatty acid synthase (FAS) or polyketide synthase (PKS) proteins (type I), or as a small monomeric protein in the system for fatty acid biosynthesis in bacteria and plastids (type II FAS) and in the dissociated PKSs (type II PKS) (White et al., 2005; Lai et al., 2006; Smith, 2006; Byers and Gong, 2007; Chan et al., 2009). In the bacterial fatty acid 
biosynthetic pathway, ACP is a small (molecular mass $<10 \mathrm{kDa}$ ), acidic (isoelectric point $<4$ ) protein, which acts as a carrier of acyl intermediates (White et al., 2005). ACP is synthesized in the inactive apo form, which must be converted to the active holo form by a holo-ACP synthase (AcpS) that transfers the $4^{\prime}$-phosphopantetheine from coenzyme CoA (CoA) to a conserved serine residue of apo-ACP (Lambalot et al., 1996; Flugel et al., 2000). The fatty acyl group is linked to ACP by a thioester bond, in which the thiol of the $4^{\prime}$-phosphopantetheine prosthetic group in ACP is condensed with the carboxyl group of the fatty acid (White et al., 2005).

In Escherichia coli, there is a single and absolutely essential ACP, which is involved not only in the synthesis of fatty acyl chains but also in their transfer during phospholipid, lipid A or haemolysin synthesis (Byers and Gong, 2007). However, bacteria with complex metabolism often have additional ACPs or ACP homologs that are involved the synthesis of specialized fatty acids, polyketides or non-ribosomal peptides (Lai et al., 2006; Byers and Gong, 2007; Chan et al., 2009). Sinorhizobium meliloti has at least six ACPs, and four of them have been well characterized (Geiger and Lopez-Lara, 2002): AcpP for general fatty acid synthesis (López-Lara and Geiger, 2000), NodF for the synthesis of polyunsaturated fatty acids in nodulation factors (Demont et al., 1993; Demont-Caulet et al., 1999), AcpXL for the synthesis and transfer of 27-hydroxyoctacosanoic acid during rhizobial lipid A synthesis (Brozek et al., 1996; Haag et al., 2011), and RkpF, which participates in the synthesis of rhizobial capsular polysaccharides (Epple et al., 1998). The remaining two ACP homologs, SMc01553 and SMb20651, also have been identified as ACP-like proteins, but their functions need further investigation (Ramos-Vega et al., 2009; Davila-Martinez et al., 2010).

Pseudomonas aeruginosa is an opportunistic pathogen in humans, and is associated with infections in burn patients and in the respiratory tracts of patients with cystic fibrosis (Driscoll et al., 2007; Moore and Flaws, 2011). In P. aeruginosa, fatty acids play a multifaceted role in both maintaining bacterial viability and virulence (Hoang et al., 2002; Zhu and Rock, 2008; Yuan et al., 2012). Aside from being compulsory components of membrane phospholipids, fatty acids are utilized by multiple primary and secondary metabolic pathways in $P$. aeruginosa, including the formation of lipopolysaccharides (LPS) (Raetz and Whitfield, 2002), the synthesis of two important metabolic enzyme cofactors, lipoate and biotin (Spalding and Prigge, 2010), the production of three acylated quorum-sensing (QS) signal molecules-pseudomonas quinolone signal (PQS), N-(3oxodo-decanoyl)-L-homoserine lactone (3-oxo- $\mathrm{C}_{12}$-HSL), and $\mathrm{N}$-butanoyl-L-homoserine lactone ( $\mathrm{C}_{4}$-HSL) (Jimenez et al., 2012) - and the assembly of two virulence factors, siderophore pyoverdine (Hannauer et al., 2012) and rhamnolipids (Zhu and Rock, 2008). Therefore, the fatty acid biosynthetic pathway in $P$. aeruginosa is believed to be an attractive target for the development of new antimicrobial agents.

The $P$. aeruginosa genome contains three open reading frames annotated as encoding ACP (Murugan et al., 2010). PA2966 (now named acpP1) encodes AcpP1, which is found in the fatty acid biosynthetic gene cluster (Kutchma et al., 1999). PA1869 (now named acpP2) is the second ACP-encoding gene, and
PA3334 (now named acpP3), which encodes the third ACP, is located in a large gene cluster, and is considered to be involved in the synthesis of an unknown secondary metabolite (Quadri et al., 1999). Although, in vitro, these three apo-ACPs have been successfully phosphopantetheinylated by E. coli holo-ACP synthase and all holo-forms of these three proteins can be acylated using chemical methods (Raychaudhuri et al., 2005), there is little known about their functions in vivo. Previous studies showed that $P$. aeruginosa LasI used AcpP1 as a substrate to produce 3-oxo- $\mathrm{C}_{12}$-HSL (Hoang et al., 2002) and that AcpP2 was a good substrate for RhlI in the synthesis of $\mathrm{C}_{4}$-HSL (Raychaudhuri et al., 2005), which suggests that these three ACPs may have different functions in P. aeruginosa. However, there is a lack of in vivo evidence to support the above hypothesis.

In this study, we constructed $P$. aeruginosa acpP2 and acpP3 mutant strains by insertion of a gentamicin resistance cassette into their ORFs, respectively, and an acpP1 mutant strain by replacement of the $a c p P 1$ gene with $E$. coli acpP. We analyzed various exo-products (including rhamnolipids, siderophore, and three quorum-sensing signal molecules) and tested a selection of virulence factors in the above mutant strains. The results confirmed that only AcpP1 plays roles in the production of these exo-products and virulence factors.

\section{MATERIALS AND METHODS}

The supply sources were as follows: malonyl-CoA, acetyl-CoA, fatty acids, cerulenin, NADH, NADPH, and antibiotics were obtained from Sigma (St. Louis, MO, United States). Takara Biotechnology (Dalian, China) provided molecular biology reagents. Novagen (Madison, WI, United States) provided pET vectors. Invitrogen (Carlsbad, CA, United States) provided the $\mathrm{Ni}^{2+}$-agarose column. Bio-Rad (Hercules, CA, United States) provided the Quick Start Bradford dye reagent. All other reagents were of the highest available quality.

\section{Bacterial Strains, Plasmids, and Growth Media}

The E. coli K-12 strains, $P$. aeruginosa strains, and plasmids used in this study are listed in Supplementary Table S1. LuriaBertani (LB) medium was used as the enrichment medium for growth of E. coli and P. aeruginosa. Chromobacterium violaceum CV026 and Agrobacterium tumefaciens NTL4 (pZLR4) were grown on $\mathrm{LB}$ medium at $28^{\circ} \mathrm{C}$. Antibiotics were used at the following concentrations (in micrograms per milliliter): sodium ampicillin, 100; kanamycin sulfate, 30; chloramphenicol, 30; and gentamicin, 10 (for E. coli) or 100 (for P. aeruginosa). L-Arabinose was used at a final concentration of $0.01 \%$. Isopropyl- $\beta-D-$ thiogalactoside (IPTG) was used at a final concentration of $1 \mathrm{mM}$, and 5-bromo-4-chloro-3-indolyl- $\beta$-D-galactoside (X-Gal) was used at a concentration of $20 \mu \mathrm{g} / \mathrm{mL}$.

\section{Recombinant DNA Techniques and Construction of Plasmids}

To clone the $P$. aeruginosa acpP1, acpP2, and acpP3 genes, genomic DNA was extracted from $P$. aeruginosa strain PAO1 
using the Takara DNA extraction kit. The PCR products, amplified from strain PAO1 genomic DNA using Pfu DNA polymerase and the primers listed in Supplementary Table S2, were digested by NdeI and HindIII and inserted into pBAD24m (Zhu et al., 2010) between the same sites to produce plasmids: pBAD24m-acpP1, pBAD24m-acpP2, and pBAD24m-acpP3. The acpPs genes were confirmed by sequencing by Shanghai Sangon, Inc. (Shanghai, China).

To produce plasmids pCD4 or pCD5, the PCR fragments that were amplified from plasmids pBAD24m-acpP1 or pBAD24m-acpP2, using the primers listed in Supplementary Table S2, were digested with XbaI and HindIII and cloned into pET-28 (b) at the same sites. The acpP3 fragment of pBAD24m-acpP3 digested by NdeI and HindIII was cloned between the NdeI and HindIII sites of pET-30 (a) to yield plasmid pCD6. To construct plasmids pCD8, pCD9, and pCD10, the plasmids $\mathrm{pCD} 4, \mathrm{pCD} 5$, and $\mathrm{pCD} 6$ were digested with $\mathrm{XbaI}$ and HindIII, respectively, and the DNA fragments were purified and inserted into the same sites of pBluescript $\mathrm{SK}(+)$, respectively. To yield plasmids $\mathrm{pCD} 1, \mathrm{pCD} 2$, and $\mathrm{pCD} 3$, the PCR fragments were amplified from plasmids pCD8, pCD9, or pCD10 using the primers listed in Supplementary Table S2, respectively, and then digested with double restriction enzymes, for which the sites had been designed into the primer sequences. After purification, the fragments were ligated with the vector pTac85 (Marsh, 1986), and they were digested with the same restriction enzymes, respectively.

\section{Expression and Purification of Plasmid-Encoded Proteins}

The pET-28b-derived plasmids pCD4, pCD5, and pCD6 were introduced into E. coli strain BL21 (DE3), and the respective proteins, PaAcpP1, PaAcpP2, and PaAcpP3, were expressed at high levels and purified as described previously (Thomas and Cronan, 2005). The plasmids pYFJ84 and pCD15 were introduced into E. coli strain BL21 (DE3) for expression of Vibrio harveyi AasS and P. aeruginosa PcpS proteins. The AasS and PcpS proteins were also purified as described previously (Barekzi et al., 2004; Jiang et al., 2006).

\section{Phosphopantetheinylatation and Acylation of $P$. aeruginosa ACPs}

The form of the ACPs was detected by measuring their migration pattern using conformationally sensitive gel electrophoresis. Briefly, the standard assay mixtures contained, in a volume of $40 \mu \mathrm{L}, 0.1 \mathrm{M}$ Tris- $\mathrm{HCl}$ ( $\mathrm{pH} 8.8$ ); $1 \mathrm{mM} \mathrm{CoA}, 25 \mathrm{mM} \mathrm{MgCl}$, $2 \mathrm{mM}$ DTT, $100 \mu \mathrm{M}$ P. aeruginosa ACPs, and $2 \mu \mathrm{g}$ P. aeruginosa PcpS. The mixture was pre-incubated at $37^{\circ} \mathrm{C}$ for $1 \mathrm{~h}$. The reaction products were resolved by conformationally sensitive gel electrophoresis on $17.5 \%$ polyacrylamide gels containing $0.5 \mathrm{M}$ urea optimized for separation. For purification of $P$. aeruginosa holo-ACPs, $2 \mathrm{~mL}$ of the above reaction mixture was used and incubated at $37^{\circ} \mathrm{C}$ for $4 \mathrm{~h}$. The purification of holo-ACPs was carried out according to the methods described previously (Zhao et al., 2005). Briefly, two volumes of acetone were added to the reaction mixture, and the mixture was allowed to precipitate overnight at $-20^{\circ} \mathrm{C}$. The precipitates were pelleted at $20,000 \times g$ for $20 \mathrm{~min}$ at $4^{\circ} \mathrm{C}$ and then washed twice with three volumes of acetone. The pellet was air-dried and re-suspended in $200 \mu \mathrm{L}$ Tris- $\mathrm{HCl}(20 \mathrm{mM}, \mathrm{pH} 7.4)$, and the suspension was centrifuged at $20,000 \times g$ for $30 \mathrm{~min}$ at $4^{\circ} \mathrm{C}$. The supernatants were collected and stored at $-80^{\circ} \mathrm{C}$. Their molecular masses were determined by matrix-assisted laser desorption/ionization (MALDI)-time-of-flight (TOF)-mass spectrometry (MS) (Bruker Autoflex III; Freemont, CA, United States) according to the methods described previously (Mao et al., 2015).

For acylation of $P$. aeruginosa holo-ACPs, we used the protocols described by Jiang et al. (2006). The reaction mixture contained, in a volume of $40 \mu \mathrm{L}, 10 \mathrm{mM}$ ATP, $20 \mathrm{mM} \mathrm{MgSO}_{4}$, $0.1 \mathrm{M}$ Tris- $\mathrm{HCl}$ ( $\mathrm{pH}$ 7.8), $1 \mathrm{mM}$ dithiothreitol, $0.1 \mathrm{mg} P$. aeruginosa holo-ACPs, $0.5 \mathrm{mM}$ octanoic acid and $4 \mu \mathrm{g} V$. harveyi AasS. The reaction mixtures were incubated at $37^{\circ} \mathrm{C}$ for $2 \mathrm{~h}$ and resolved by conformationally sensitive gel electrophoresis on $17.5 \%$ polyacrylamide gels containing $2.5 \mathrm{M}$ urea optimized for the separation.

\section{Reconstruction of $\boldsymbol{P}$. aeruginosa Fatty Acid Synthesis in Vitro}

Cell-free extracts of $P$. aeruginosa were prepared according the processes described previously (Hoang and Schweizer, 1999). The exponentially growing cells $\left(\mathrm{OD}_{600}\right.$ of 0.8 to 1.0$)$ in $100 \mathrm{~mL} \mathrm{LB}$ medium were harvested by centrifugation and then suspended and washed in $2 \mathrm{~mL} 0.1 \mathrm{M}$ sodium phosphate buffer ( $\mathrm{pH} 6.5$ ). Cell lysates were prepared by passing the cell suspensions three times through a French pressure cell $\left(19,000 \mathrm{lb} / \mathrm{in}^{2}\right)$. Cell debris was removed by ultracentrifugation for $1 \mathrm{~h}$ at $200,000 \times g$, and the supernatants were saved as cell extracts. The protein concentration of the cell extract was determined using the Quick Start Bradford dye reagent. The fatty acid synthetic reaction mixtures included, in a volume of $100 \mu \mathrm{L}, 0.1 \mathrm{M}$ Tris- $\mathrm{HCl}$ (pH 7.8), $1 \mathrm{mM}$ dithiothreitol, $100 \mu \mathrm{M}$ P. aeruginosa holoACPs (AcpP1, AcpP2, or AcpP3), $100 \mu \mathrm{M}$ NADH, $100 \mu \mathrm{M}$ NADPH, $100 \mu \mathrm{M}$ malonyl-CoA, $100 \mu \mathrm{M}$ acetyl-CoA and $5 \mathrm{mg}$ $P$. aeruginosa cell-free extract. Reactions were incubated at $37^{\circ} \mathrm{C}$ for $2 \mathrm{~h}$. Analysis of the ACP thioesters was performed by conformationally sensitive gel electrophoresis on $17.5 \%$ polyacrylamide gels containing $2.5 \mathrm{M}$ urea optimized for the separation. Cerulenin was added to the reaction mixtures at a final concentration of $100 \mu \mathrm{M}$ when needed.

\section{Mutation of $P$. aeruginosa acpPs Genes}

To disrupt the $P$. aeruginosa acpPs genes, suicide plasmids were constructed as follows. The 1.3-kb DNA fragment containing acpP1 amplified from $P$. aeruginosa genomic DNA using the primers listed in Supplementary Table S2 was digested with BamHI and HindIII and cloned between the same sites of pBluescript SK $(+)$ to produce pCD16. A gentamicin resistance cassette amplified from p34s-Gm (Dennis and Zylstra, 1998) using the primers listed in Supplementary Table S2 was digested by EcoRI and inserted into the same site of pCD16 to produce pCD17. Subsequently, the BamHI-HindIII fragment from pCD17 was inserted between the same sites of $\mathrm{pK} 18$ mobsacB 
(Schafer et al., 1994) to yield the suicide plasmid pCD18. A similar method was used to construct the suicide plasmids pCD21 and pCD25. All these plasmids were introduced into $P$. aeruginosa PAO1 via conjugal transfer from E. coli S17-1 (Supplementary Figure S1A). Single-crossover integrants into the strain PAO1 chromosome were selected by chloramphenicol and gentamicin resistance. Cultures grown from the integrant colonies were plated onto LB medium containing $10 \%$ sucrose and gentamicin in order to select for the loss of the vector sequences from the PAO1 chromosome. The successful construction of the designed mutations was evaluated by PCR analysis using the primers listed in Supplementary Table S2. Two single mutant strains were obtained: PA-A2 $\left(\operatorname{acpP} 2:: \mathrm{Gm}^{\mathrm{r}}\right)$ and PA-A3 $\left(\operatorname{acpP} 3:: \mathrm{Gm}^{\mathrm{r}}\right)$ (Supplementary Figures S1B,C), but no acpP1 insertion mutant was obtained.

To replace $P$. aeruginosa acpP1 with $E$. coli acpP, the DNA fragments acpP1up, EcacpP and acpP1down were amplified using the primers listed in Supplementary Table S2. The above three DNA fragments were ligated by overlap PCR and cloned into the T-vector, pMD19, to produce pCD29. The BamHI-HindIII fragment from pCD29 was cloned between the same sites of pK18mobsacB to yield plasmid pCD30. The gentamicin resistance cassette of p34s-Gm, digested by BamHI, was inserted into the same site of pCD30 to produce plasmid pCD31. Using similar procedures, the replacement strain PA-A1 (acpP1::EcacpP) was obtained (Supplementary Figure S3).

To obtain double or triple P. aeruginosa acpPs mutant strains, we also constructed plasmid pCD22, in which the gentamicin resistance cassette in plasmid pCD 21 was replaced with a tetracycline resistance cassette (Dennis and Zylstra, 1998). Using similar procedures to those described above, on a background of PA-A1, we obtained the double mutant strains PA-A12 $\left(\right.$ acpP1::EcacpP acpP2::Gm ${ }^{\mathrm{r}}$ ) (Supplementary Figure S4A) and PA-A13 ( $а с p P 1:: E c a c p P$ acpP3:: $\mathrm{Gm}^{\mathrm{r}}$ ) (Supplementary Figure S4B). On a background of PA-A3, we obtained the double mutant strain PA-A23 (acpP2::Tc ${ }^{\mathrm{r}}$ acpP3:: $\mathrm{Gm}^{\mathrm{r}}$ ) (Supplementary Figure $\mathrm{S} 4 \mathrm{C}$ ), and on a background of $\mathrm{PA}-\mathrm{A} 23$, we produced a triple mutant strain, PA-A123 (acpP1::EcacpP acpP2::Tc ${ }^{\mathrm{r}}$ acpP3::Gm ${ }^{\mathrm{r}}$ ) (Supplementary Figure S4D).

To confirm the mutant strains, all acpP allelic genes were sequenced by Shanghai Sangon, Inc. (Shanghai, China).

\section{Analysis of Phospholipid Composition}

The cultures were grown aerobically at $37^{\circ} \mathrm{C}$ in $\mathrm{LB}$ medium overnight. Cells were harvested and washed three times with sterile water. Fatty acid methyl esters were synthesized and extracted as described previously (Stead, 1989). Briefly, cellular lipids were saponified by the addition of $2 \mathrm{~mL}$ of sodium hydroxide/methanol solution at $100^{\circ} \mathrm{C}$ for $40 \mathrm{~min}$ with shaking ( $800 \mathrm{rpm})$. The fatty acids were then methylated by the addition of $4 \mathrm{~mL}$ of hydrochloric acid/methanol solution at $80^{\circ} \mathrm{C}$ for $30 \mathrm{~min}$, and immediately cooled to below $20^{\circ} \mathrm{C}$. Fatty acid methyl esters were obtained by three extractions, each with $1 \mathrm{~mL}$ of petroleum ether. The solvent was removed under a stream of nitrogen, and the residue was dissolved in $100 \mu \mathrm{L}$ of hexane. The crude extract was passed through a $0.22 \mu \mathrm{m}$ Mini-star filter unit, and $2 \mu \mathrm{L}$ of the extract was analyzed by gas chromatographymass spectrometry (GC-MS).

\section{Motility Assay}

The motility assay was carried out as described previously (Huang et al., 2016). The swarming motility of $P$. aeruginosa was investigated using the following media: $0.45 \%$ tryptone, $0.13 \%$ yeast extract, $0.5 \%$ agarose, $0.22 \% \mathrm{NaCl}$, and $0.5 \%$ agar. The plates were air-dried for 5-10 min before use. The bacterial cells $\left(\mathrm{OD}_{600}\right.$ to 0.8$)$ were gently inoculated using a toothpick at the center of the media surface, and the plates were incubated at $30^{\circ} \mathrm{C}$ for $24 \mathrm{~h}$. The twitching motility assay was performed using the following media: $1 \%$ tryptone, $0.5 \%$ yeast extract, $0.5 \% \mathrm{NaCl}$, and $1 \%$ agarose. The cells were stabbed into the bottom of a Petri dish containing each of the above media using a toothpick, and incubated at $37^{\circ} \mathrm{C}$ for $20 \mathrm{~h}$. The movement of the colony on the interface between the medium and the dish was observed.

\section{Rhamnolipid Identification}

Rhamnolipids were extracted as previously described (Huang et al., 2016). Briefly, cells were removed from PPGAS $\left(\mathrm{NH}_{4} \mathrm{Cl}\right.$ $20 \mathrm{mM}, \mathrm{KCl} 20$ mM, Tris- $\mathrm{HCl}$ pH 7.2120 mM, MgSO $1.6 \mathrm{mM}$, glucose $0.5 \%$, and peptone $1.0 \%$ ) cultures grown at $30^{\circ} \mathrm{C}$ for $24 \mathrm{~h}$ by centrifugation $(10 \mathrm{~min}$ at $6,000 \times g)$, and the supernatant was acidified to $\mathrm{pH} 2$ with concentrated $\mathrm{HCl}$. Equal volumes $(1 \mathrm{~mL})$ of acidified supernatant and chloroform:methanol (2:1) were mixed and vortexed for $1 \mathrm{~min}$, and the lower organic phase was collected after centrifugation $(10 \mathrm{~min}$ at $10,000 \times g)$. The extraction was repeated, and the pooled organic phases were evaporated to dryness, re-suspended in $1 \mathrm{~mL}$ methanol, filtered through a $0.45-\mu \mathrm{m}$ membrane, evaporated to dryness, and re-suspended in $20 \mu \mathrm{L}$ methanol. The samples were analyzed by thin-layer chromatography (TLC) using silica $60 \mathrm{~F}_{254}$ plates (Merck, Darmstadt, Germany) with a mobile phase consisting of chloroform:methanol:acetic acid (65:15:2, v/v/v). Orcinol reagent dissolved in $15 \% \mathrm{H}_{2} \mathrm{SO}_{4}$ at a final concentration of $2 \%$ was used to visualize rhamnolipid spots at $100^{\circ} \mathrm{C}$ for $2-5 \mathrm{~min}$. The concentration of rhamnolipid was determined by measuring the concentration of rhamnose with the sulfuric acid-anthrone reagent $(0.2 \%$ anthrone, $85 \%$ sulfuric acid) method, using rhamnose sugar as the standard, at $620 \mathrm{~nm}$. To identify the fatty acid component of the rhamnolipid, rhamnolipids were extracted from $5 \mathrm{~mL}$ supernatant using the same procedures as described above. Fatty acid methyl esters were synthesized using rhamnolipid and extracted as described previously (Zhu et al., 2010). Briefly, the rhamnolipids were dissolved in $1.2 \mathrm{~mL}$ dry methanol, and $0.2 \mathrm{~mL}$ of $25 \%$ (vol/vol) sodium methoxide was added. After the solution was allowed to stand for $15 \mathrm{~min}$ at room temperature, $1.2 \mathrm{~mL}$ of $2 \mathrm{M} \mathrm{HCl}$ was added, and the fatty acid methyl esters were obtained by three extractions each with $1.2 \mathrm{~mL}$ of petroleum ether. The solvent was removed under a stream of nitrogen, and the residue was analyzed by measuring the formation of hydroxylacyl-ACP. The standard mixture contained $0.1 \mathrm{M}$ sodium phosphate ( $\mathrm{pH} 7.0$ ), $10 \mathrm{mM}$ ATP, $20 \mathrm{mM} \mathrm{MgSO}_{4}$, $1 \mathrm{mM}$ DTT, $100 \mu \mathrm{M}$ E. coli ACP, $1 \mu \mathrm{g} / \mu \mathrm{L}$ His-tagged $V$. harveyi AasS purified from $E$. coli and fatty acid methyl esters obtained from rhamnolipid. The mixture was incubated at $37^{\circ} \mathrm{C}$ for $1 \mathrm{~h}$ 
and resolved by conformationally sensitive gel electrophoresis on $17.5 \%$ polyacrylamide gels containing $2.5 \mathrm{M}$ urea optimized for the separation.

\section{Extraction and Assay of Quorum-Sensing Signal Molecules}

Extraction of quorum-sensing signal molecules was performed as described previously (Yuan et al., 2012). P. aeruginosa cells were grown in LB to the early stationary phase, and cells were removed from $10 \mathrm{~mL}$ growth medium by centrifugation at $12,000 \times g$ for $15 \mathrm{~min}$ at $4^{\circ} \mathrm{C}$. Quorum-sensing signal molecules were twice extracted from $10 \mathrm{~mL}$ of supernatant for each sample, using either an equal volume of ethyl acetate for homoserine lactones (HSL) or an equal volume of acidified ethyl acetate for 2-heptyl3-hydroxy-4(1H)-quinolone (PQS). The organic extracts were concentrated to dryness using a nitrogen bubbler, and the residue was resuspended in $100 \mu \mathrm{L}$ of ethyl acetate (HSL) or methanol (PQS). For determination of AHL-like molecules with short acyl chains, the biosensor $C$. violaceum $\mathrm{CV} 026$ was utilized (McClean et al., 1997). A. tumefaciens NTL4 (pZLR4) was utilized to detect AHLs with long acyl chains (Cha et al., 1998). HPLC analysis was carried out with an HPLC series (Agilent Technologies, Palo Alto, CA, United States) equipped with an Elite C18 column (4.6 $\mathrm{mm} \times 250 \mathrm{~mm}, 5 \mu \mathrm{m}$ particle size) that was maintained at $45^{\circ} \mathrm{C}$, with a UV detector set at $250 \mathrm{~nm}$. The mobile phase comprised $0.1 \%$ formic acid in water $(\mathrm{A})$ and $0.1 \%$ formic acid in acetonitrile (B) (Ortori et al., 2007). The flow rate was $1 \mathrm{~mL} / \mathrm{min}$. The elution conditions were as follows: 0 min $35 \% \mathrm{~B}$, linear gradient to $60 \% \mathrm{~B}$ for $10 \mathrm{~min}$ and then a linear gradient from 60 to $95 \%$ B over $5 \mathrm{~min}$, then $5 \mathrm{~min} 95 \% \mathrm{~B}$ and then ramped back to the starting conditions in $9 \mathrm{~min}$. The column was re-equilibrated for a total of $5 \mathrm{~min}$. A $10-\mu \mathrm{L}$ volume was injected onto the column. PQS content was assessed by normal-phase thin-layer chromatography (TLC) on activated silica 60 F254 plates (Merck) according the method described previously (Yuan et al., 2012). Authentic acyl- and 3-oxo-acyl-HSL, and PQS (Sigma) were used as standards.

\section{LasA and LasB Activity Assay}

Pseudomonas aeruginosa cells were grown in $\mathrm{LB}$ at $37^{\circ} \mathrm{C}$ for $12 \mathrm{~h}$, and cells were removed from $10 \mathrm{~mL}$ growth medium by centrifugation at $5,000 \times g$ for $10 \mathrm{~min}$ at $4^{\circ} \mathrm{C}$. LasA protease activity was determined by measuring the ability of $P$. aeruginosa culture supernatants to lyse boiled Staphylococcus aureus RN4200 cells, as described previously (Huang et al., 2016). A 30-mL overnight culture of $S$. aureus grown in tryptic soy broth was placed in a boiling water bath for $10 \mathrm{~min}$ and then centrifuged for $10 \mathrm{~min}$ at $10,000 \times g$. The resulting pellet was re-suspended in $10 \mathrm{mM} \mathrm{Na}_{2} \mathrm{HPO}_{4}$ (pH 7.5) and adjusted to an $\mathrm{OD}_{600}$ of 0.9. A $100-\mu \mathrm{L}$ aliquot of bacterial supernatant was then added to $900 \mu \mathrm{L}$ of $S$. aureus suspension, and the $\mathrm{OD}_{600}$ was determined after $5,10,15,20,25,30,35,40,45,60,75,90$, and $105 \mathrm{~min}$. LasB protease activity in $P$. aeruginosa culture supernatants was determined using the elastin Congo red assay (Yuan et al., 2012). After a 3-h digestion period at $37^{\circ} \mathrm{C}(100 \mathrm{mM}$ Tris, $1 \mathrm{mM} \mathrm{CaCl}$ [pH 7.5] with $20 \mathrm{mg}$ of elastin Congo red substrate [Sigma] plus $100 \mu \mathrm{L}$ of spent supernatant), suspensions were clarified by centrifugation $(16,000 \times g, 5 \mathrm{~min}$, RT), and the absorbance at $495 \mathrm{~nm}$ was measured to determine the amount of liberated dye.

\section{Pyocyanin Quantitation Assay}

The pyocyanin assay is based on the absorbance of pyocyanin at $520 \mathrm{~nm}$ in acidic solution (Essar et al., 1990). A 5-mL sample of culture grown in LB was extracted with $3 \mathrm{~mL}$ of chloroform and then re-extracted into $1 \mathrm{~mL}$ of $0.2 \mathrm{~N} \mathrm{HCl}$ to give a pink to deep red solution. The absorbance of this solution was measured at $520 \mathrm{~nm}$. Concentrations, expressed as $\mu \mathrm{g}$ of pyocyanin produced per $\mathrm{mL}$ of culture supernatant, were determined by multiplying the optical density at $520 \mathrm{~nm}\left(\mathrm{OD}_{520}\right)$ by 17.072 .

\section{Siderophore Secretion Assay}

The CAS-LB plates were prepared according to a modification of the published protocol (Yuan et al., 2012). A 10× blue dye solution consisting of $1 \mathrm{mM}$ chrome azurol S (CAS) (Acros), $2 \mathrm{mM}$ cetyltrimethylammonium bromide, and $500 \mu \mathrm{M} \mathrm{FeCl}_{3}$ $6 \mathrm{H}_{2} \mathrm{O}$ was sterilized by autoclaving, and then $10 \mathrm{~mL}$ was added to $100 \mathrm{~mL}$ of molten LB Miller agar (1.5\%). Plates were allowed to solidify and dried at room temperature for $1 \mathrm{~h}$ before streaking cultures containing each strain to be tested.

\section{Statistical Analysis}

An analysis of variance for the experimental datasets was performed using JMP software version 5.0 (SAS Institute Inc., Cary, NC, United States). Significant effects of treatment were determined by the $F$-value $(P=0.05)$. When a significant $F$-test was obtained, a separation of means was accomplished by Fisher's protected LSD (least significant difference) at $P=0.05$.

\section{RESULTS}

\section{Only $P$. aeruginosa AcpP1 Functions in Fatty Acid Biosynthesis}

In order to test whether $P$. aeruginosa acpP1, acp P2, and acpP3 function in fatty acid biosynthesis, these genes were inserted into the IPTG-inducible vector pTac85 (Marsh, 1986) to yield plasmids pCD1 (carrying Pa acpP1), pCD2 (carrying Pa acpP2), and $\mathrm{pCD} 13$ (carrying $\mathrm{Pa}$ acpP3), respectively. The plasmids were then used to transform E. coli strain CY1877, a strain in which the chromosomal acpP gene had been deleted and replaced with a chloramphenicol resistance cassette. The deletion replacement event was done in the presence of an ampicillinresistant pBAD24-derived plasmid expressing the E. coli acpP gene to allow survival of the acpP deletion strain. Growth of strain CY1877 was dependent on the addition of arabinose because transcription of the $a c p P$ gene was from a vector $a r a B A D$ promoter. Only E. coli CY1877 carrying the plasmid encoding acpP1 ( $\mathrm{pCD} 1$ ) grew on LB medium in the presence of IPTG and the absence of arabinose, whereas derivatives of strain CY1877 carrying empty vector, or plasmids encoding acpP2 or acpP3 failed to grow under this condition (Figure 1A). P. aeruginosa acpP1 complemented the E. coli acpP mutation, showing that 


\section{A}

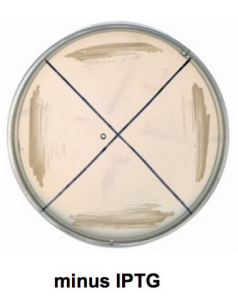

B

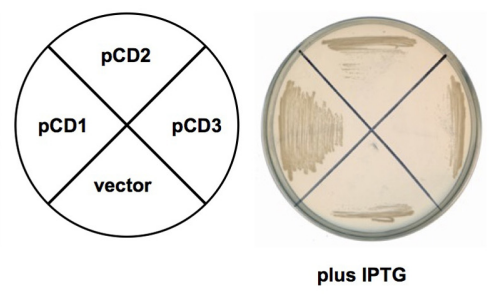

C
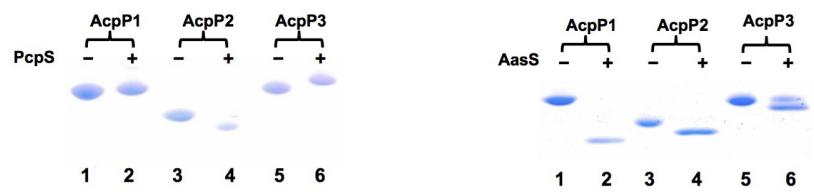

D

Cell free extracts

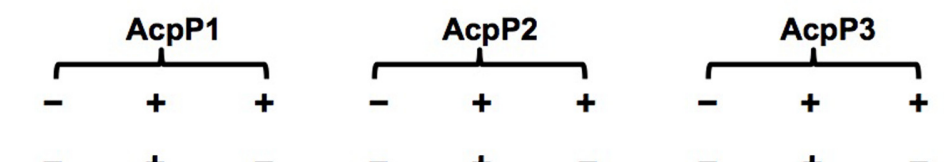

Cerulenin

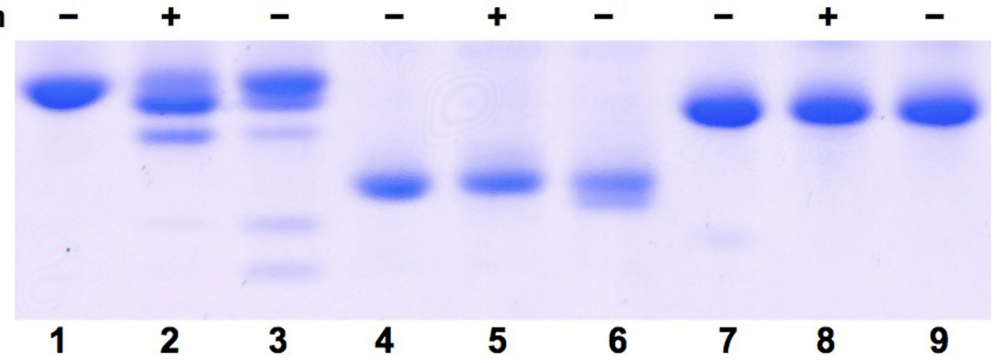

FIGURE 1 | Identification of Pseudomonas aeruginosa AcpPs in vivo and in vitro. (A) Complementation of Escherichia coli acpP mutant strain CY1877 with $P$. aeruginosa acpP genes. E. coli acpP mutant strain carried plasmids pCD1, pCD2, pCD3 and empty vector, and was grown on LB plates. pCD1, plasmid pTac 85 carrying acpP1; pCD2, plasmid pTac 85 carrying acpP2; pCD3, plasmid pTac 85 carrying acpP3; vector, pTac 85. (B) The P. aeruginosa apo-AcpPs were phosphopantetheinylated by P. aeruginosa PcpS in vitro. P. aeruginosa AcpPs were purified from an E. coli strain according to the procedures described in section "Materials and Methods." The reaction mixture containing each kind of AcpP, CoA and $P$. aeruginosa PcpS was incubated for 2 h before being resolved by conformationally sensitive gel electrophoresis as described in section "Materials and Methods." (C) The P. aeruginosa holo-AcpPs were acylated by Vibrio harveyi AasS in vitro. The reaction mixture containing each kind of AcpP, ATP, octanoic acid and $V$. harveyi AasS was incubated for $2 \mathrm{~h}$ before being resolved by conformationally sensitive gel electrophoresis as described in section "Materials and Methods". (D) The P. aeruginosa AcpPs were used as substrates in fatty acid synthesis catalyzed by $P$. aeruginosa cell-free extracts. The reaction mixture containing each kind of AcpP, acetyl-CoA, malonyl-CoA, NADPH, NADH and cell-free extracts was incubated for $2 \mathrm{~h}$ before being resolved by conformationally sensitive gel electrophoresis as described in section "Materials and Methods."

P. aeruginosa AcpP1 can function as an ACP and replace functional E. coli ACP. To examine further whether P. aeruginosa AcpP2 and AcpP3 are involved in fatty acid synthesis, these ACPs were overexpressed and purified as described in the section "Materials and Methods." First, phosphopantetheinylatation of these three ACPs was tested. As expected, after incubation with $P$. aeruginosa phosphopantetheinyl transferase (PcpS) and CoA, the migration of AcpP1 did not change significantly, suggesting that $P$. aeruginosa AcpP1 may be activated from apo-ACP to holo-ACP by E. coli holo-ACP synthase (AcpS) (Figure 1B), which is consistent with the finding that acpP1 complemented the E. coli acpP mutation. To confirm the above observation, we analyzed AcpP1, which was purified from E. coli cells directly or from phosphopantetheinylatation with $P$. aeruginosa PcpS, by MALDI-TOF-MS (Supplementary Figure S5). The data showed that AcpP1 purified from E. coli cells includes apo- and holo- forms of ACPs, while after phosphopantetheinylatation with $P$. aeruginosa PcpS, the AcpP1 all became holo-AcpP1
(Supplementary Figures S5A,B). However, both AcpP2 and AcpP3 showed different migration rates after incubation with PcpS and CoA (Figure 1B), indicating that the purified AcpP2 and AcpP3 proteins from E. coli were apo-ACPs, and that AcpP2 and AcpP3 could not be activated properly in E. coli from apo-ACP to holo-ACP by E. coli AcpS, which were confirmed by analysis with MALDI-TOF-MS (Supplementary Figures S5C-F). We also tested whether these ACPs can be acylated by $V$. harveyi acyl-ACP synthetase (AasS). After incubation of these holoACPs with octanoic acid, ATP and AasS, all reaction mixtures produced new bands on native gels, showing that all these holo-ACPs could be acylated by AasS (Figure 1C). Finally, whether these ACPs can be used as substrates during fatty acid synthesis by $P$. aeruginosa in vitro was examined. As expected, $P$. aeruginosa AcpP1 was a good substrate for fatty acid synthesis. The reaction mixture containing holo-AcpP1, malonyl-CoA, acetyl-CoA, NADH, NADPH and $P$. aeruginosa cell-free extract produced short-chain acyl-ACPs in the presence 
TABLE 1 | Fatty acid composition of total lipid extracts from Pseudomonas aeruginosa strains ${ }^{a}$.

\begin{tabular}{lrrrr}
\hline Fatty acid (\%) & \multicolumn{4}{c}{$\boldsymbol{P}$ aeruginosa strains } \\
\cline { 2 - 5 } & \multicolumn{1}{c}{ PAO1 } & \multicolumn{1}{c}{ PA-A1 } & \multicolumn{1}{c}{ PA-A2 } & \multicolumn{1}{c}{ PA-A3 } \\
\hline$n-\mathrm{C}_{10: 0}-3-\mathrm{OH}^{\mathrm{b}}$ & $3.78 \pm 0.69$ & $6.01 \pm 0.84$ & $4.62 \pm 1.99$ & $5.40 \pm 0.68$ \\
$n-\mathrm{C}_{12: 0}-3-\mathrm{OH}$ & $6.60 \pm 0.76$ & $6.59 \pm 0.87$ & $6.51 \pm 0.23$ & $6.79 \pm 1.02$ \\
$\mathrm{n}-\mathrm{C}_{16: 1}$ & $11.26 \pm 0.50$ & $10.40 \pm 1.51$ & $9.87 \pm 1.66$ & $10.83 \pm 1.50$ \\
$\mathrm{n}-\mathrm{C}_{16: 0}$ & $34.49 \pm 0.36$ & $34.90 \pm 0.37$ & $36.10 \pm 0.69$ & $36.30 \pm 0.83$ \\
$\mathrm{n}-\mathrm{C}_{18: 1}$ & $35.85 \pm 1.91$ & $36.12 \pm 2.02$ & $36.08 \pm 0.68$ & $34.13 \pm 1.16$ \\
$\mathrm{n}-\mathrm{C}_{18: 0}$ & $8.01 \pm 0.35$ & $5.97 \pm 0.72$ & $6.81 \pm 0.79$ & $6.55 \pm 0.91$ \\
\hline
\end{tabular}

${ }^{a}$ Cells were grown in $L B$ medium for $12 h$ at $37^{\circ} \mathrm{C}$. Total lipids were extracted and transesterified to fatty acid methyl esters, and products identified by GC-MS. Values are percentages of total fatty acids and are means \pm standard deviations of three independent experiments. ${ }^{\mathrm{b}} n-\mathrm{C}_{10: 0} 3-\mathrm{OH}$, 3-hydroxyldecanoic acid; $n-C_{10: 0}$ 3-OH, 3-hydroxyldodecanoic acid; $n-C_{16: 1}$, cis-9-hexadecenoic acid; $n-C_{16: 0}$, hexadecanoic acid; $n-C_{18: 1}$, cis-11-octadecenoic acid; $n-C_{18: 0}$, octadecanoic acid.

of cerulenin, an antibiotic that inhibits the activity of long chain 3-keto-acyl-ACP synthases. In the absence of cerulenin, the reaction mixture produced various long-chain acyl-ACPs (Figure 1D). However, P. aeruginosa AcpP2 and AcpP3 were poor substrates for fatty acid synthesis in vitro: only the reaction mixture containing AcpP2 produced a trace amount of shortchain acyl-ACPs in the absence of cerulenin (Figure 1D), but the reaction mixture containing AcpP3 did not produce any acyl-ACPs. All these results imply that $P$. aeruginosa AcpP2 and AcpP3 are not involved in fatty acid synthesis in $P$. aeruginosa.

\section{acpP1 Is Essential for $P$. aeruginosa Growth}

To examine further the physiological functions of the three $P$. aeruginosa ACP homologs, we attempted to inactivate each of the putative acpP genes by allelic replacement. Three pK18mobsacB-borne suicide plasmids used to insert a gentamicin resistance cassette into $a c p P 1, a c p P 2$, or $a c p P 3$ genes were constructed and yielded pCD18, pCD21, and pCD25, respectively (Supplementary Figure S1). These plasmids were introduced into $P$. aeruginosa $\mathrm{PAO} 1$ via conjugal transfer from E. coli S17-1 (Supplementary Figure S1). After negative selection, mediated by Bacillus subtilis sacB, and identification by colony PCR using the primers listed in Supplementary Table S2, two single mutant strains were obtained: PA-A2 $\left(\operatorname{acpP} 2:: \mathrm{Gm}^{\mathrm{r}}\right)$ and PA-A3 (acpP3::Gm ${ }^{\mathrm{r}}$ ) (Supplementary Figures S1A,B), but no acpP1 insertion mutant was obtained, which indicates that $P$. aeruginosa acp $P 1$ seems to be an essential gene.

To confirm this finding, two new suicide plasmids, pCD28 (Supplementary Figure S2) and pCD31 (Supplementary Figure S3A), were constructed. The plasmid pCD28 was used to delete $a c p P 1$, while $\mathrm{pCD} 31$ was used to replace $P$. aeruginosa acpP1 with $E$. coli acpP. Both plasmids were introduced into $P$. aeruginosa PAO1 by conjugation. Cultures grown from the single-integrant colonies were plated onto LB medium containing sucrose. Colonies that were sensitive to gentamicin were analyzed by PCR using the primers listed in Supplementary Table S2. Unfortunately, no acp P1 deletion strain was obtained (Supplementary Figure S2). However, we obtained a replacement mutation strain, PA-A1 (acp 1::EcacpP), in which the $P$. aeruginosa acp $P 1$ gene was replaced with the $E$. coli acp $P$ gene (Supplementary Figures S3B,C). These results indicated that acpP1 is essential for the growth of $P$. aeruginosa.

Using similar procedures, we also constructed three double mutants, PA-12 (acpP1::EcacpP, acpP2::Gm $\left.\mathrm{Gm}^{\mathrm{r}}\right)$ (Supplementary Figure S4A), PA-13 (acpP1::EcacpP, acpP3::Gm ${ }^{\mathrm{r}}$ ) (Supplementary Figure S4B), and PA-23 (acpP2:: $\mathrm{Tc}^{\mathrm{r}}$, acpP3:: $\mathrm{Gm}^{\mathrm{r}}$ ) (Supplementary Figure $\mathrm{S} 4 \mathrm{C}$ ), and one triple mutant, PA-123 (acpP1::EcacpP, acpP2:: $\mathrm{Tc}^{\mathrm{r}}, \quad$ acpP3:: $\mathrm{Gm}^{\mathrm{r}}$ ) (Supplementary Figure S4D). Next, the growth of these mutant strains was tested. All mutant strains were viable and grew as well as wild type strain PAO1 (data not shown).

\section{Replacement of acpP1 with E. coli acpP Reduced the Ability of $P$. aeruginosa to Produce Quorum-Sensing Signals and Rhamnolipids}

To investigate further the functions of the three ACP homologs in fatty acid synthesis in $P$. aeruginosa, the fatty acid composition of the acpPs mutant strains grown in LB medium was first determined by gas chromatography-mass spectrometry (GC-MS). The fatty acid profiles of all the mutant strains were almost the same and were not distinct from that of wild type strain PAO1 (Table 1 and Supplementary Table S3). This indicated that E. coli ACP protein may replace functions of $P$. aeruginosa AcpP1, and that AcpP2 and AcpP3 may not play roles in fatty acid synthesis.

In $P$. aeruginosa, fatty acids are not only the compulsory components of membrane phospholipids, but are also utilized to produce exo-products, including three acylated quorum-sensing signal molecules, rhamnolipids and siderophore pyoverdine (Hoang et al., 2002; Zhu and Rock, 2008; Yuan et al., 2012). To confirm the above hypothesis and to determine whether AcpP2 or AcpP3 is specified to synthesize these exo-products, the production of these exo-products in $P$. aeruginosa acpPs mutant strains was examined.

First, the rhl QS signal molecule, $N$-butanoyl-L-homoserine lactone $\left(\mathrm{C}_{4}\right.$-HSL), produced by $P$. aeruginosa acpPs mutant strains was tested using the C. violaceum reporter strain CV026, which produces a purple halo in response to acyl-HSLs. The purple halo around mutant strains PA-A2, PA-A3, and PA-A23 was as large as that around wild type strain PAO1 (Figure 2A). The amount of $\mathrm{C}_{4}$-HSL produced by PA-A2, PA-A3, or PA-A23 was also determined by high performance liquid chromatography (HPLC), which showed that all these mutant strains produced almost the same amount of $\mathrm{C}_{4}$-HSL (about $4.1 \mu \mathrm{M}$ ), which was similar to the amount produced by the PAO1 strain (Figure 2B). However, the purple halo around mutant strain PA-A1 was weak and small in comparison with that of wild type strain PAO1 (Figure 2C). The HPLC analysis showed that the amount of $\mathrm{C}_{4}$-HSL produced by PA-A1 (about $2.4 \mu \mathrm{M}$ ) was significantly lower than that produced by the PAO1 strain (about $4.1 \mu \mathrm{M}$ ) $(P<0.01)$ (Figure 2C). In contrast, the double mutant strains, 
A
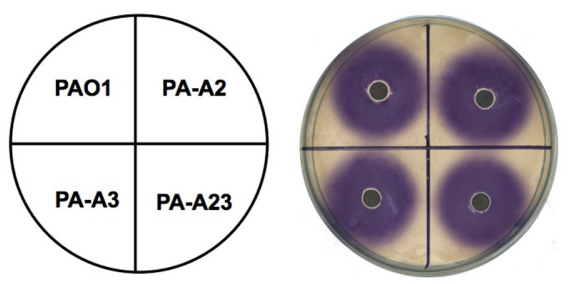

C

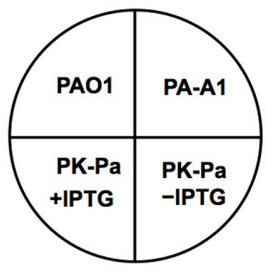

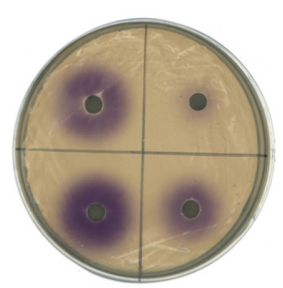

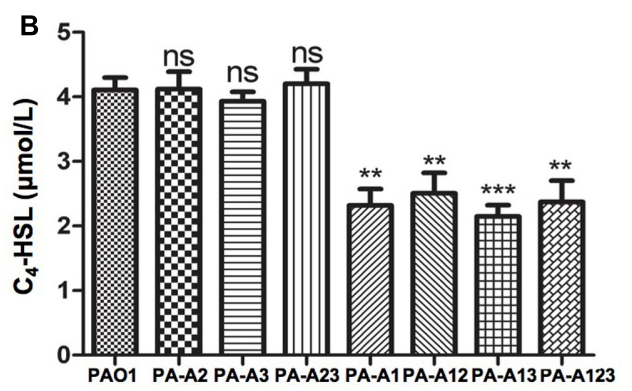

FIGURE 2 | Detection of $\mathrm{C}_{4}-\mathrm{HSL}$ signals produced by $P$. aeruginosa mutant strains. (A) Analysis of the $\mathrm{C}_{4}-\mathrm{HSL}$ signals produced by $P$. aeruginosa acpP2 and acpP3 mutant strains using Chromobacterium violaceum reporter strain CV026. (B) Quantitative analysis of the $\mathrm{C}_{4}$-HSL signal produced by $P$. aeruginosa acpPS mutant strains using high performance liquid chromatography (HPLC). (C) Analysis of the $\mathrm{C}_{4}-\mathrm{HSL}$ signals produced by $P$. aeruginosa acpP1 mutant strains using C. violaceum reporter strain CV026. The acylated HSL fraction was extracted from the supernatants of stationary-phase cultures grown in $L B$ broth at $37^{\circ} \mathrm{C}$ for $12 \mathrm{~h}$. PAO1, P. aeruginosa wild type strain; PA-A2, P. aeruginosa acpP2::Gm ${ }^{r}$ single mutant strain; PA-A3, P. aeruginosa acpP3::Gm ${ }^{r}$ single mutant strain; PA-A23, P. aeruginosa acp2::Tc ${ }^{r}$ acp3::Gm ${ }^{r}$ double mutant strain; PA-A1, P. aeruginosa acp1::Ec acpP single mutant strain; PA-A12, P. aeruginosa acp 1::Ec acpP acp2::Gm ${ }^{r}$ double mutant strain; PA-A13, P. aeruginosa acp 1::Ec acpP acp3::Gmr double mutant strain; PA-A123, P. aeruginosa acp1::Ec acpP acp2::Tcr acp3::Gm triple mutant strain; $\mathrm{PK}-\mathrm{Pa}, P$. aeruginosa acp 1::Ec acpP single mutant strain carrying plasmid, pSRKPa, encoding wild type $P$. aeruginosa acpP1 gene. Data are the mean \pm standard deviation of triplicate measurements. Pair-wise comparisons were made between wild-type strain PAO1 and each mutant strain by Student's $t$-test. ${ }^{* *}$ Highly significant difference, $P<0.01$. ${ }^{* *}$ Significant difference, $P<0.05$. ns, no significant difference, $P>0.1$.

PA-A12 and PA-A13, and the triple mutant, PA-123, produced a similar amount of $\mathrm{C}_{4}$-HSL to the PA-A1 strain (Figure 2B). However, expression of the wild-type $P$. aeruginosa acpP1 gene in mutant strain PA-A1 increased the production of $\mathrm{C}_{4}$-HSL, and under IPTG induction the amount of $\mathrm{C}_{4}$-HSL was restored to the level of the wild type strain (Figure 2C).

The las QS signal molecule, $N$-(3-oxododecanoyl)-Lhomoserine lactone (3-oxo- $\left.\mathrm{C}_{12}-\mathrm{HSL}\right)$, produced by these mutant strains was also investigated using the reporter strain A. tumefaciens NL4(pZLR4) and HPLC. The data showed that replacement of acpP1 with $E$. coli acp $P$ reduced the ability of $P$. aeruginosa to produce 3 -oxo- $\mathrm{C}_{12}-\mathrm{HSL}$ (Figures $\mathbf{3 A}, \mathbf{B}$ ), but mutation of acpP2 or acpP3 did not affect the production of the las QS signal molecule (Figures 3B,C).

We analyzed the levels of PQS in supernatant extracts of the mutant strains by thin-layer chromatography (TLC). The level of PQS was reduced $40 \%$ in the supernatant extract of mutant PA-A1 in comparison with that produced by wild type strain PAO1. However, disruption of acpP2 or acpP3 did not cause reduction of the amount of $\mathrm{PQS}$ produced by $P$. aeruginosa strains (data not shown).

Rhamnolipids are secreted surfactant glycolipids formed by acylation of L-rhamnose using 3-hydroxydecanoyl-ACP (Zhu and Rock, 2008). Therefore, the levels of rhamnolipids produced by $P$. aeruginosa mutant strains were examined. First, TLC was used to analyze the rhamnolipids produced by
$P$. aeruginosa strains. The amount of rhamnolipids in acpP1 mutant strain PA-A1 was decreased, but complementation with plasmid pSRK-Pa, in which the wild-type acpP1 gene was cloned into pSRK-Km, restored the ability to produce rhamnolipids to the level of that in wild type strain PAO1 (Figure 4A). Quantification of rhamnolipids by colorimetric detection of rhamnose showed a twofold decrease in the acpP1 mutant PA-A1, while the amount of rhamnolipids produced by complemented strain PK-Pa (PA-A1/pSRKPa) was up to $80 \%$ of that of the wild type strain PAO1 (Supplementary Figure S6). We also analyzed the 3-hydroxyacids composition of the rhamnolipids produced by acpP1 mutant strains. The data showed that, although the same fatty acid species were present in PA-A1 as in the wild type PAO1, the 3-hydroxyacids were quantitatively reduced among the rhamnolipids produced by PA-A1 (Figure 4B). Next, we investigated the amount of rhamnolipids produced by $P$. aeruginosa acp $P 2$ or acp $P 3$ mutant strains. The level of rhamnolipids produced by PA-A2, PA$\mathrm{A} 3$, or PA-A23 was almost the same as that in strain PAO1. In strain PA-A1, disruption of a single acpP2 or single acpP3, or disruption of both acpP2 and acpP3 genes, did not cause the reduction in rhamnolipids seen following replacement of $P$. aeruginosa acpP1 with the E. coli acpP gene (data not shown).

Pyoverdine is the dominant siderophore of $P$. aeruginosa and is assembled from tetradecanoyl-ACP (Quadri et al., 1999). 


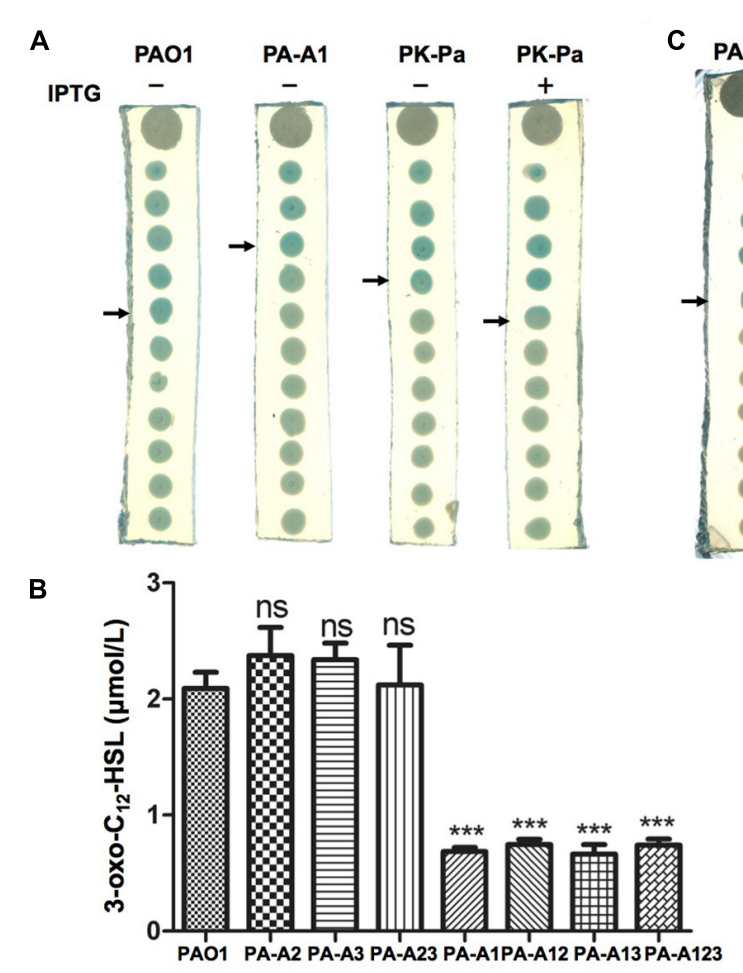

FIGURE 3 | Detection of 3-oxo- $\mathrm{C}_{12}-\mathrm{HSL}$ signals produced by $P$. aeruginosa mutant strains. (A) Analysis of the 3-oxo- $\mathrm{C}_{12}-\mathrm{HSL}$ signals produced by $P$. aeruginosa acpP1 mutant strains using Agrobacterium tumefaciens reporter strain NTL4 (pZLR4). (B) Quantitative analysis of the 3-oxo- $\mathrm{C}_{12}-\mathrm{HSL}_{\mathrm{S}}$ signals produced by $P$. aeruginosa acpPs mutant strains using high performance liquid chromatography (HPLC). (C) Analysis of the 3-oxo- $\mathrm{C}_{12}-\mathrm{HSL}$ signals produced by $P$. aeruginosa acpP2 and acpP3 mutant strains using $A$. tumefaciens reporter strain NTL4 (pZLR4). The acylated HSL fraction was extracted from the supernatants of stationary-phase cultures grown in LB broth at $37^{\circ} \mathrm{C}$ for $12 \mathrm{~h}$. PAO1, P. aeruginosa wild type strain; PA-A2, P. aeruginosa acpP2::Gm ${ }^{r}$ single mutant strain; PA-A3, $P$. aeruginosa acpP3::Gm ${ }^{r}$ single mutant strain; PA-A23, P. aeruginosa acp2::Tc ${ }^{r}$ acp3::Gm ${ }^{r}$ double mutant strain; PA-A1, P. aeruginosa acp 1::Ec acpP single mutant strain; PA-A12, P. aeruginosa acp 1::Ec acpP acp2::Gm double mutant strain; PA-A13, P. aeruginosa acp 1::Ec acpP acp3::Gm ${ }^{r}$ double mutant strain; PA-A123, P. aeruginosa acp1::Ec acpP acp2::Tc ${ }^{r}$ acp3::Gm ${ }^{r}$ triple mutant strain; PK-Pa, $P$. aeruginosa acp 1::Ec acpP single mutant strain carrying plasmid, pSRKPa, encoding wild type $P$. aeruginosa acpP1 gene. The arrow indicates the diffusion front of $3-\mathrm{oxo}_{-} \mathrm{C}_{12}-\mathrm{HSL}$. Data are the mean \pm standard deviation of triplicate measurements. Pair-wise comparisons were made between wild-type strain PAO1 and each mutant strain by Student's $t$-test. ***Highly significant difference, $P<0.01$. ** Significant difference, $P<0.05$.

Secretion of pyoverdine by $P$. aeruginosa acpPs mutant strains was examined by culture on LB-chrome azurol $S$ (CAS) indicator plates. Zones around the cultures that change from blue to yellow-orange indicate where the siderophores have sequestered $\mathrm{Fe}^{3+}$ away from the blue CAS-Fe ${ }^{3+}$ complex. Both wild type strain PAO1 and mutant strain PA-A1 generated similar prominent clear zones, which suggested that replacement of $a c p P 1$ with $E$. coli acpP did not affect siderophore secretion (Figure 4C). Moreover, the yellow-orange halo around mutant strains PA-A2, PA-A3, and PA-A23 was as large as that around strain PAO1. In addition, on a background of strain PA-A1, mutation of acpP2 or acpP3 did not reduce siderophore secretion by $P$. aeruginosa (data not shown).

On the basis of the above results, we conclude that neither acpP2 nor acpP3 is involved in fatty acid synthesis in $P$. aeruginosa and that they have no function in the production of various exo-products. Furthermore, although E. coli ACP replaces the function of $P$. aeruginosa AcpP1 in fatty acid synthesis, $P$. aeruginosa AcpP1 is required for the production of some exo-products, including three acylated quorum-sensing signal molecules and rhamnolipids.

\section{Replacement of acpP1 with E. coli acpP Abolished $P$. aeruginosa Swarming Motility}

The QS systems, including rhl, las, and PQS, not only control the production of many virulence factors by $P$. aeruginosa (such as LasA/LasB, rhamnolipid, procyanin, and others), but are also involved in the regulation of $P$. aeruginosa motility (Jimenez et al., 2012). Replacement of $a c p P 1$ by E. coli acpP caused $P$. aeruginosa to reduce the production of QS signal molecules. Therefore, the mutant strain PA-A1 was expected to have reduced production of some virulence factors and attenuated motility. To confirm this, we first tested the production of LasA, LasB, and procyanin. LasA protease activity was determined by measuring the ability of $P$. aeruginosa culture supernatants to lyse boiled $S$. aureus cells. The data showed that the activity of LasA in mutant strain PA-A1 was much lower than that 

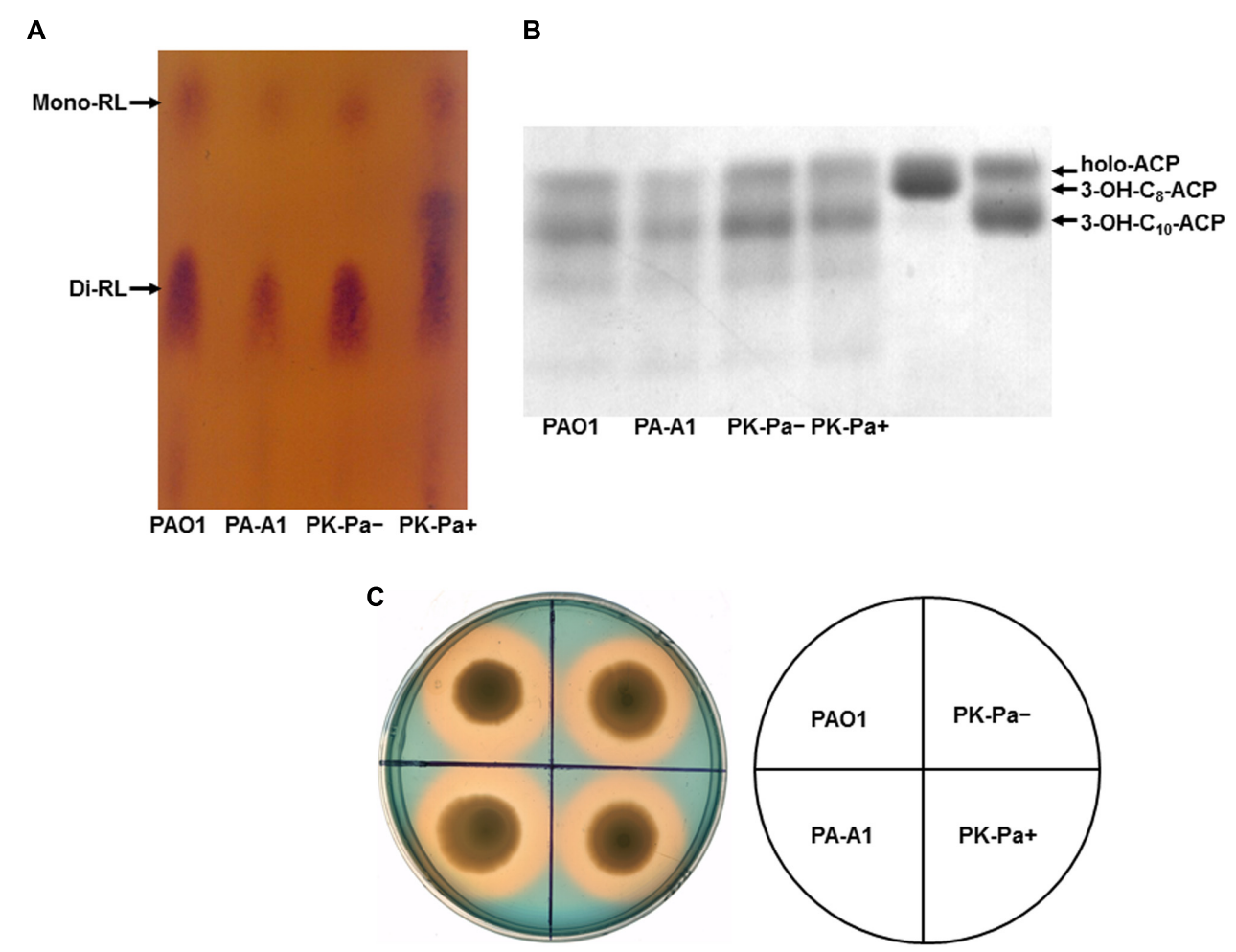

FIGURE 4 | Characterization of rhamnolipid production and siderophore secretion by $P$. aeruginosa acpP1 mutant strains. (A) Analysis of rhamnolipids produced by acpP1 mutant strains using thin-layer chromatography (TLC). (B) Composition of 3-hydroxyacids in rhamnolipids produced by acpP1 mutant strains.

(C) Siderophore secretion in $P$. aeruginosa acpP1 mutant strains. PAO1, $P$. aeruginosa wild type strain; PA-A1, $P$. aeruginosa acp1::Ec acpP single mutant strain; PK-Pa ${ }^{-}, P$. aeruginosa acp 1::Ec acpP single mutant strain carrying plasmid, pSRKPa, encoding wild type $P$. aeruginosa acpP1 gene under no IPTG induction; PK-Pa+, complementary strain PK-Pa under IPTG induction.

in wild type strain PAO1, but complementation strain PK-Pa (PA-A1/pSRK-Pa) increased LasA to the level of that in wild type strain PAO1 (Figure 5A). The elastase proteolytic activity (LasB) was measured using the elastin Congo red assay. In comparison with that in wild type strain PAO1, the activity of LasB was decreased twofold in the PA-A1 strain, while the activity of LasB in complemented strain PK-Pa (PA-A1/pSRK-Pa) was up to $80 \%$ of that in wild type strain PAO1 (data not shown). However, procyanin production by mutant strain PA-A1 was significantly increased $(P<0.005)$ in comparison with wild type strain PAO1, and plasmid pSRK-Pa did not restore the ability of strain PA-A1 to produce procyanin to the level of that in the wild type strain, indicating that other factors may be involved in the regulation of the production of procyanin in mutant strain PA-A1 (Figure 5B).

We tested three types of motility: swimming, swarming, and twitching, in mutant strain PA-A1. The swimming pattern of mutant stain PA-A1 was almost the same as that of wild type strain PAO1, which suggested that replacement of acpP1 with E. coli acp $P$ did not affect the swimming motility of $P$. aeruginosa (data not shown). However, replacement of acpP1 by E. coli acpP reduced the twitching ability of $P$. aeruginosa. The twitching pattern of mutant PA-A1 was much smaller than that of the PAO1 strain (Figure 6A). We also tested the effect of mutation of acpP2 or acpP3 on twitching motility. The data showed that neither single mutation of $a c p P 2$ or $a c p P 3$ nor double mutation of acpP2 and acpP3 affected $P$. aeruginosa twitching motility (Figures 6A,B).

Next, we investigated the swarming motility of mutant strain PA-A1. Replacement of acpP1 by E. coli acpP caused $P$. aeruginosa swarming to fail. On semisolid plates (containing $0.5 \%$ agar), wild type strain PAO1 formed a typical swarming pattern, while mutant strain PA-A1 only formed a small circular lawn (Figure 7A). However, when complemented with pSRK-Pa, the ability of PA-A1 to form a typical swarming pattern was restored (Figure 7B). We also noticed that mutant strains PA-A2, PA-A3, and PA-A23 could form a typical swarming pattern, indicating that mutation of $a c p P 2$ or $a c p P 3$ did not affect $P$. aeruginosa swarming motility (Figures 7A,B). Because replacement of acpP1 by $E$. coli acpP causes $P$. aeruginosa to reduce the production of QS signal molecules, we wondered if exogenous addition of AHLs signals could restore the ability of mutant strain PA-A1 to swarm on semisolid plates. To test this hypothesis, we added $2 \mu \mathrm{M} \mathrm{C}_{4}$-HSL or 3-oxo- $\mathrm{C}_{12}$-HSL to semisolid plates. After $24 \mathrm{~h}$ of incubation, on both types of semisolid plate, the swarming motility of mutant strain PA-A1 was restored (Figure 7C), even though the swarming patterns were still smaller than that of the wild type strain PAO1. This indicated that reduction of the AHLs 


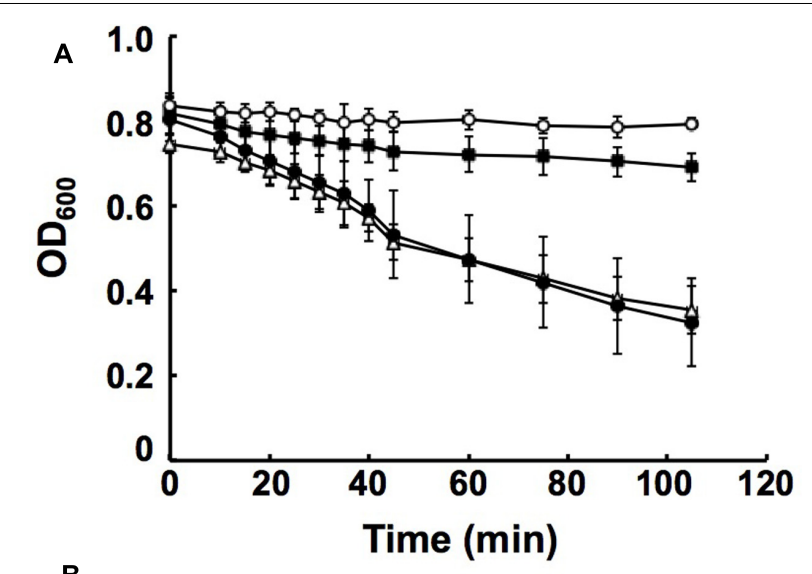

B

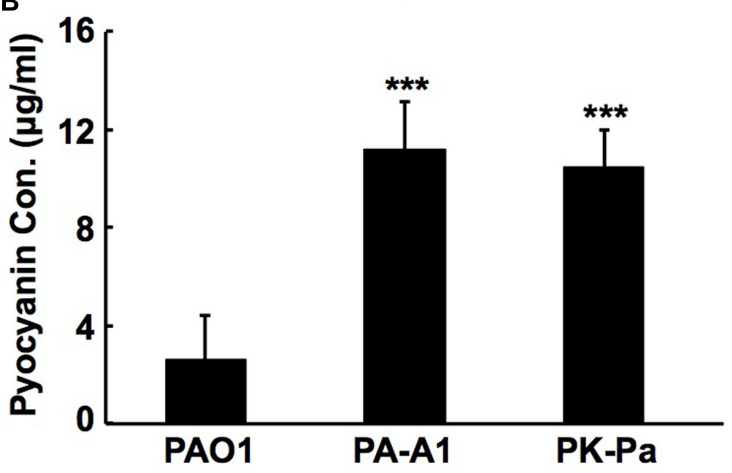

FIGURE 5 | Analysis of LasA activities and pyocyanin production in $P$. aeruginosa acpP1 mutant strains. (A) LasA protease activities in culture supernatants of $P$. aeruginosa strains. Empty circle indicates boiled Staphylococcus aureus cells; filled square indicates boiled $S$. aureus cells containing culture supernatants of $P$. aeruginosa mutant strain PA-A1; filled circle indicates boiled $S$. aureus cells containing culture supernatants of

$P$. aeruginosa PAO1; empty triangle indicates boiled $S$. aureus cells containing culture supernatants of complementary strain PK-Pa. (B) Pyocyanin in culture supernatants of $P$. aeruginosa strains. Data are the mean \pm standard deviation of triplicate measurements. Pair-wise comparisons were made between wild type strain PAO1 and mutant strain PA-A1 or complementary strain PK-Pa by Student's $t$-test. ***Highly significant difference, $P<0.01$.

signal was the main factor that caused $P$. aeruginosa to fail to swarm.

\section{DISCUSSION}

The $P$. aeruginosa genome contains three open reading frames, PA2966, PA1869, and PA3334, that encode putative ACP: AcpP1, AcpP2, and AcpP3, respectively. Alignments of $P$. aeruginosa AcpPs with the E. coli ACP showed that AcpP1, AcpP2, and AcpP3 share 87, 45, and 30\% identical residues with E. coli $\mathrm{ACP}$, respectively, and the serine residue that is presumed to be phosphopantetheinylated is conserved among these AcpPs (Raychaudhuri et al., 2005). Our in vitro assays showed that these apo-ACPs were successfully phosphopantetheinylated by $P$. aeruginosa $\mathrm{PcpS}$ and that all holo-forms of these proteins could be acylated by $V$. harveyi AasS, which suggested that all these proteins may be ACP. However, only AcpP1 could be used as

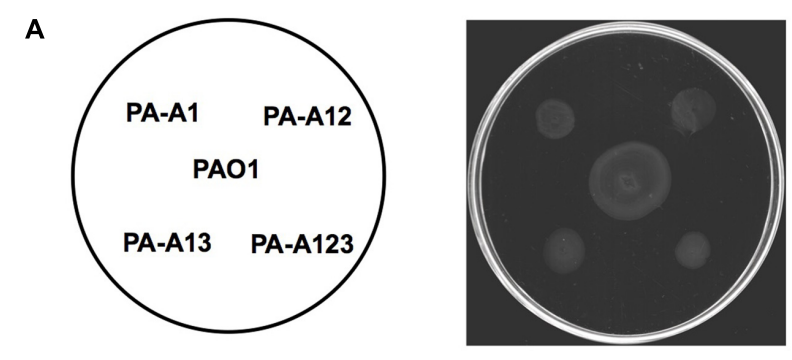

B
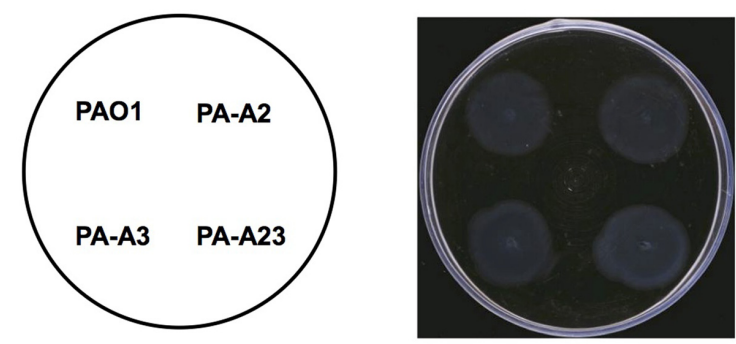

FIGURE 6 | Twitching motility of $P$. aeruginosa strains. (A) Twitching motility patterns of $P$. aeruginosa acpP1 mutant strains after incubation at $37^{\circ} \mathrm{C}$ for 24 h. PAO1, P. aeruginosa wild type strain; PA-A1, P. aeruginosa acp1::Ec acpP single mutant strain; PA-A12, P. aeruginosa acp 1::Ec acpP acp2::Gm ${ }^{r}$ double mutant strain; PA-A13, P. aeruginosa acp 1::Ec acpP acp3::Gm ${ }^{r}$ double mutant strain; PA-A123, P. aeruginosa acp 1::Ec acpP acp2:: $\mathrm{Tc}^{r}$ acp3:: Gmr triple mutant strain. (B) Twitching motility patterns of $P$. aeruginosa acpPs mutant strains after incubation at $37^{\circ} \mathrm{C}$ for $24 \mathrm{~h}$. PAO1, P. aeruginosa wild type strain; PA-A2, P. aeruginosa acpP2::Gm ${ }^{r}$ single mutant strain; PA-A3, P. aeruginosa acpP3::Gm single mutant strain; PA-A23, P. aeruginosa acp2:: $\mathrm{Tc}^{r}$ acp3::Gm ${ }^{r}$ double mutant strain.

a substrate for the synthesis of fatty acids in vitro, catalyzed by $P$. aeruginosa cell-free extracts, and only acp 1 could restore growth in the E. coli acpP mutant strain CY1877. Moreover, acp $P 1$ is essential for the growth of $P$. aeruginosa and cannot be deleted, but disruption of acp $P 2$ or acp $P 3$ in $P$. aeruginosa mutant strains allowed them to grow as well as the wild type strain, and mutation of the acpP2 or acpP3 gene did not cause changes in the fatty acid composition in $P$. aeruginosa cells. These findings confirm that only $P$. aeruginosa AcpP1 functions in fatty acid biosynthesis, and that acpP2 and acpP3 do not play roles in the fatty acid synthetic pathway.

Replacement of $P$. aeruginosa acp $P 1$ with $E$. coli acp $P$ did not affect $P$. aeruginosa growth, and the fatty acid composition of this mutant strain was almost the same as that of the wild type strain, which suggests that E. coli ACP may be a good substrate for $P$. aeruginosa fatty acid synthetic enzymes. However, the production of some exo-products, including three acylated quorum-sensing signal molecules $\left(\mathrm{C}_{4}\right.$-HSL, 3-oxo- $\mathrm{C}_{12}$-HSL, and PQS) and rhamnolipids, was reduced. It is well known that the precursors of these products come from intermediates in the $P$. aeruginosa fatty acid synthetic pathway (Hoang et al., 2002). However, the E. coli strain possesses no enzymes involved in the production of these exo-products and therefore does not produce the above exo-products. Thus, even though E. coli ACP can be used as a good substrate for 


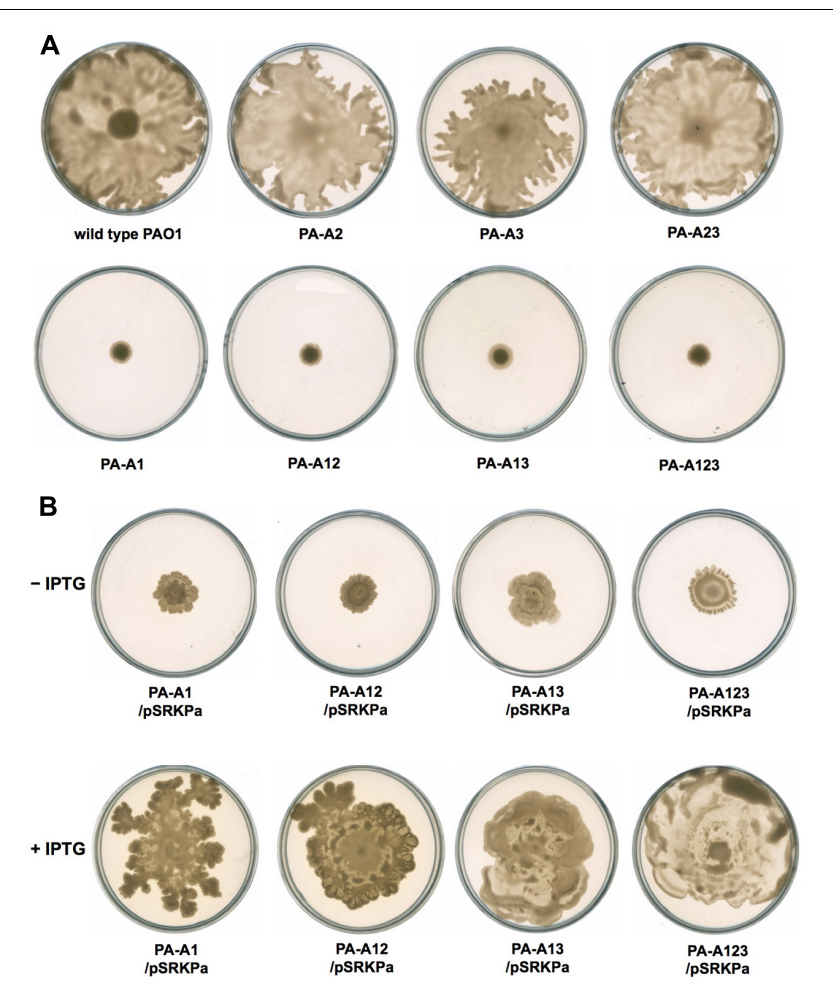

C

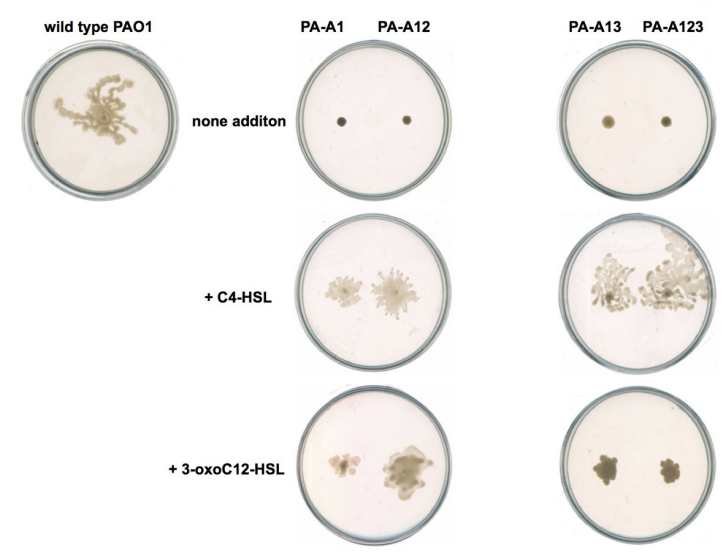

FIGURE $7 \mid$ Swarming motility of $P$. aeruginosa strains. (A) Swarming motility patterns of $P$. aeruginosa acpPs mutant strains. (B) Swarming motility patterns of $P$. aeruginosa acpP1 mutant strains carried plasmid pSRK-Pa. (C) Effect of exogenous AHLs on swarming motility of $P$. aeruginosa acpP1 mutant strains. PAO1, P. aeruginosa wild type strain; PA-A1, P. aeruginosa acp 1::Ec acpP single mutant strain; PA-A2, P. aeruginosa acpP2::Gmr single mutant strain; $\mathrm{PA}-\mathrm{A} 3, P$. aeruginosa acpP3::Gm${ }^{r}$ single mutant strain; PA-A12, P. aeruginosa acp 1::Ec acpP acp2::Gm double mutant strain; PA-A13,

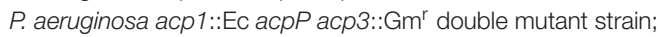

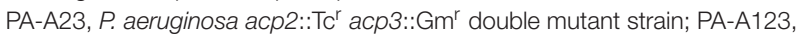
P. aeruginosa acp 1::Ec acpP acp2:: $\mathrm{Tc}^{r}$ acp3::Gm triple mutant strain.

$P$. aeruginosa fatty acid synthetic enzymes to produce acyl-ACP intermediates, $P$. aeruginosa enzymes that are responsible for the production of the above exo-products fail to recognize intermediates carried by E. coli ACP as a good substrate, which causes reduced production of these exo-products in $P$. aeruginosa.

Replacement of acpP1 with E. coli acpP also abolished $P$. aeruginosa swarming motility. However, addition of exogenous $\mathrm{C}_{4}$-HSL or 3 -oxo- $\mathrm{C}_{12}$-HSL could restore the swarming motility of the $P$. aeruginosa mutant PA-A1 strain, which was consistent with the reduction in the amount of $\mathrm{C}_{4}$-HSL or 3-oxo- $\mathrm{C}_{12}$-HSL produced by mutant PA-A1. A similar phenomenon was also observed when we studied the $P$. aeruginosa $f a b V$ mutant: the $f a b V$ mutant showed reduced production of $\mathrm{C}_{4}$-HSL and 3-oxo- $\mathrm{C}_{12}$-HSL signals, and failed to swarm, but addition of exogenous $\mathrm{C}_{4}$-HSL or 3-oxo- $\mathrm{C}_{12}-\mathrm{HSL}$ restored the swarming motility of the $f a b V$ mutant strain (Huang et al., 2016). These findings suggest that an insufficient number of QS signals was the main factor that caused P. aeruginosa to fail to swarm, and that the QS system plays an important role in $P$. aeruginosa swarming motility.

$P$. aeruginosa produces two siderophores, pyoverdine, and pyochelin, which are involved in iron acquisition and are required for virulence (Moore and Flaws, 2011). Replacement of acpP1 with E. coli acpP did not affect the ability of $P$. aeruginosa to secrete siderophores. This indicated that $P$. aeruginosa ACPs are not involved in the synthesis of these two siderophores. Indeed, pyoverdine and pyochelin are produced by non-ribosomal peptide synthases (Quadri et al., 1999; Barekzi et al., 2004). For pyoverdine, the associated carrier proteins are PvdD, PvdI, and PvdJ, while pyochelin synthesis requires the PchE and PchF carrier proteins (Barekzi et al., 2004).

Quorum-sensing signaling is necessary for production of the phenazine secondary metabolite pyocyanin (Jimenez et al., 2012). However, although replacement of $a c p P 1$ with $E$. coli acpP reduced the ability of $P$. aeruginosa to produce quorum-sensing signals, the mutant strain PA-A1 showed significantly increased production of pyocyanin in comparison with the wild type strain. Recent studies have shown that an RpoS-dependent sRNA RgsA represses the expression of $P$. aeruginosa AcpP1 at the posttranscriptional level by base-pairing interactions between RgsA and acpP1 mRNA (Lu et al., 2016). That report also showed that overexpression of full-length RgsA in P. aeruginosa could increase the production of pyocyanin. Therefore, on the basis of these findings, we speculate that replacement of $\operatorname{acp} P 1$ with E. coli acp $P$ caused $P$. aeruginosa to fail to produce acpP1 mRNA, and increased free RgsA in $P$. aeruginosa cells. Under the action of RgsA, $P$. aeruginosa increased the production of pyocyanin. However, the details of the mechanism need further study.

In $P$. aeruginosa, $\mathrm{C}_{4}$-HSL, a quorum-sensing signal of the $\mathrm{Rhl}$ signaling system, is produced from $S$-adenosyl-methionine and butyryl-ACP, a process catalyzed by RhlI (Jimenez et al., 2012). Previous in vitro studies (Raychaudhuri et al., 2005) showed that AcpP2 molecules are good substrates for RhlI. However, following disruption of the acpP2 gene in the $P$. aeruginosa genome, mutant strain PA-A2 did not show a reduced level of $\mathrm{C}_{4}$-HSL. Moreover, although replacement of acpP1 with E. coli acp $P$ caused the amount of $\mathrm{C}_{4}$-HSL produced by $P$. aeruginosa to decrease, the double mutant strain PA-A12, in which acpP1 was replaced with $E$. coli acp $P$ and $a c p P 2$ was disrupted, produced the same level of $\mathrm{C}_{4}$-HSL as that produced by the single mutant 
strain PA-A1. All these findings indicate that AcpP2 is not a good substrate for RhlI in vivo. Our studies also confirmed that AcpP2 was not involved in the production of 3-oxo- $\mathrm{C}_{12}$-HSL and rhamnolipids. Therefore, the functions of AcpP2 in P. aeruginosa need further investigation.

Disruption of acpP3 did not affect growth, the fatty acid composition of phospholipids or production of some exo-products in $P$. aeruginosa. This should be expected, because acpP3 is located in a gene cluster that encodes an unknown secondary metabolite synthetic pathway (Quadri et al., 1999).

\section{AUTHOR CONTRIBUTIONS}

J-CM cloned acpPs gene, and constructed expression vectors. DC constructed acpP mutants, purified ACP proteins. Y-QW tested the production of QS signals and motility in mutant strains. W-BZ analyzed fatty acids composition of acpP strains and analyzed the function of ACPs in vitro. J-CM also participated

\section{REFERENCES}

Barekzi, N., Joshi, S., Irwin, S., Ontl, T., and Schweizer, H. P. (2004). Genetic characterization of $p c p S$, encoding the multifunctional phosphopantetheinyl transferase of Pseudomonas aeruginosa. Microbiology 150, 795-803. doi: 10.1099/mic.0.26823-0

Brozek, K. A., Carlson, R. W., and Raetz, C. R. (1996). A special acyl carrier protein for transferring long hydroxylated fatty acids to lipid A in Rhizobium. J. Biol. Chem. 271, 32126-32136. doi: 10.1074/jbc.271.50.32126

Byers, D. M., and Gong, H. (2007). Acyl carrier protein: structure-function relationships in a conserved multifunctional protein family. Biochem. Cell Biol. 85, 649-662. doi: 10.1139/O07-109

Cha, C., Gao, P., Chen, Y. C., Shaw, P. D., and Farrand, S. K. (1998). Production of acyl-homoserine lactone quorum-sensing signals by gram-negative plantassociated bacteria. Mol. Plant Microbe. Interact. 11, 1119-1129. doi: 10.1094/ MPMI.1998.11.11.1119

Chan, Y. A., Podevels, A. M., Kevany, B. M., and Thomas, M. G. (2009). Biosynthesis of polyketide synthase extender units. Nat. Prod. Rep. 26, 90-114. doi: 10.1039/B801658P

Davila-Martinez, Y., Ramos-Vega, A. L., Contreras-Martinez, S., Encarnacion, S., Geiger, O., and Lopez-Lara, I. M. (2010). SMc01553 is the sixth acyl carrier protein in Sinorhizobium meliloti 1021. Microbiology 156, 230-239. doi: 10. 1099/mic.0.033480-0

Demont, N., Debelle, F., Aurelle, H., Denarie, J., and Prome, J. C. (1993). Role of the Rhizobium meliloti nodF and nodE genes in the biosynthesis of lipooligosaccharidic nodulation factors. J. Biol. Chem. 268, 20134-20142.

Demont-Caulet, N., Maillet, F., Tailler, D., Jacquinet, J. C., Prome, J. C., Nicolaou, K. C., et al. (1999). Nodule-inducing activity of synthetic Sinorhizobium meliloti nodulation factors and related lipo-chitooligosaccharides on alfalfa. Importance of the acyl chain structure. Plant Physiol. 120, 83-92. doi: 10.1104/pp.120.1.83

Dennis, J. J., and Zylstra, G. J. (1998). Plasposons: modular self-cloning minitransposon derivatives for rapid genetic analysis of gram-negative bacterial genomes. Appl. Environ. Microbiol. 64, 2710-2715.

Driscoll, J. A., Brody, S. L., and Kollef, M. H. (2007). The epidemiology, pathogenesis and treatment of Pseudomonas aeruginosa infections. Drugs 67, 351-368. doi: 10.2165/00003495-200767030-00003

Epple, G., van der Drift, K. M., Thomas-Oates, J. E., and Geiger, O. (1998). Characterization of a novel acyl carrier protein, $\mathrm{RkpF}$, encoded by an operon involved in capsular polysaccharide biosynthesis in Sinorhizobium meliloti. J. Bacteriol. 180, 4950-4954.

Essar, D. W., Eberly, L., Hadero, A., and Crawford, I. P. (1990). Identification and characterization of genes for a second anthranilate synthase in in the design of the study and helped to draft the manuscript. $\mathrm{H}-\mathrm{HW}$ conceived of the study, and participated in its design and coordination and helped to draft the manuscript. All authors read and approved the final manuscript.

\section{ACKNOWLEDGMENTS}

This work was supported by grants from the Natural Science Foundation of Guangdong Province (2015A030312005 and 2014A030313455), the National Key Project for Basic Research of China (2015CB150600) and the National Natural Science Foundation of China (31200028 and 31671987).

\section{SUPPLEMENTARY MATERIAL}

The Supplementary Material for this article can be found online at: https://www.frontiersin.org/articles/10.3389/fmicb. 2017.02186/full\#supplementary-material

Pseudomonas aeruginosa: interchangeability of the two anthranilate synthases and evolutionary implications. J. Bacteriol. 172, 884-900. doi: 10.1128/jb.172.2. 884-900.1990

Flugel, R. S., Hwangbo, Y., Lambalot, R. H., Cronan, J. E., and Walsh, C. T. (2000). Holo-(acyl carrier protein) synthase and phosphopantetheinyl transfer in Escherichia coli. J. Biol. Chem. 275, 959-968. doi: 10.1074/jbc.275.2.959

Geiger, O., and Lopez-Lara, I. M. (2002). Rhizobial acyl carrier proteins and their roles in the formation of bacterial cell-surface components that are required for the development of nitrogen-fixing root nodules on legume hosts. FEMS Microbiol. Lett. 208, 153-162. doi: 10.1111/j.1574-6968.2002.tb11075.x

Haag, A. F., Wehmeier, S., Muszynski, A., Kerscher, B., Fletcher, V., Berry, S. H., et al. (2011). Biochemical characterization of Sinorhizobium meliloti mutants reveals gene products involved in the biosynthesis of the unusual lipid A very long-chain fatty acid. J. Biol. Chem. 286, 17455-17466. doi: 10.1074/jbc.M111. 236356

Hannauer, M., Schafer, M., Hoegy, F., Gizzi, P., Wehrung, P., Mislin, G. L., et al. (2012). Biosynthesis of the pyoverdine siderophore of Pseudomonas aeruginosa involves precursors with a myristic or a myristoleic acid chain. FEBS Lett. 586, 96-101. doi: 10.1016/j.febslet.2011.12.004

Hoang, T. T., and Schweizer, H. P. (1999). Characterization of Pseudomonas aeruginosa enoyl-acyl carrier protein reductase (FabI): a target for the antimicrobial triclosan and its role in acylated homoserine lactone synthesis. J. Bacteriol. 181, 5489-5497.

Hoang, T. T., Sullivan, S. A., Cusick, J. K., and Schweizer, H. P. (2002). Beta-ketoacyl acyl carrier protein reductase (FabG) activity of the fatty acid biosynthetic pathway is a determining factor of 3-oxo-homoserine lactone acyl chain lengths. Microbiology 148, 3849-3856. doi: 10.1099/00221287-148-123849

Huang, Y. H., Lin, J. S., Ma, J. C., and Wang, H. H. (2016). Functional characterization of triclosan-resistant enoyl-acyl-carrier protein reductase (FabV) in Pseudomonas aeruginosa. Front. Microbiol. 7:1903. doi: 10.3389/ fmicb.2016.01903

Jiang, Y., Chan, C. H., and Cronan, J. E. (2006). The soluble acyl-acyl carrier protein synthetase of Vibrio harveyi B392 is a member of the medium chain acyl-CoA synthetase family. Biochemistry 45, 10008-10019. doi: 10.1021/bi060842w

Jimenez, P. N., Koch, G., Thompson, J. A., Xavier, K. B., Cool, R. H., and Quax, W. J. (2012). The multiple signaling systems regulating virulence in Pseudomonas aeruginosa. Microbiol. Mol. Biol. Rev. 76, 46-65. doi: 10.1128/MMBR.05007-11

Kutchma, A. J., Hoang, T. T., and Schweizer, H. P. (1999). Characterization of a Pseudomonas aeruginosa fatty acid biosynthetic gene cluster: purification of acyl carrier protein (ACP) and malonyl-coenzyme A:ACP transacylase (FabD). J. Bacteriol. 181, 5498-5504. 
Lai, J. R., Koglin, A., and Walsh, C. T. (2006). Carrier protein structure and recognition in polyketide and nonribosomal peptide biosynthesis. Biochemistry 45, 14869-14879. doi: 10.1021/bi061979p

Lambalot, R. H., Gehring, A. M., Flugel, R. S., Zuber, P., LaCelle, M., Marahiel, M. A., et al. (1996). A new enzyme superfamily - the phosphopantetheinyl transferases. Chem. Biol. 3, 923-936. doi: 10.1016/S1074-5521(96)90181-7

López-Lara, I. M., and Geiger, O. (2000). Expression and purification of four different rhizobial acyl carrier proteins. Microbiology 146, 839-849. doi: 10.1099/00221287-146-4-839

Lu, P., Wang, Y., Zhang, Y., Hu, Y., Thompson, K. M., and Chen, S. (2016). RpoS-dependent sRNA RgsA regulates Fis and AcpP in Pseudomonas aeruginosa. Mol. Microbiol. 102, 244-259. doi: 10.1111/mmi.13458

Mao, Y. H., Ma, J. C., Li, F., Hu, Z., and Wang, H. H. (2015). Ralstonia solanacearum RSp0194 encodes a novel 3-keto-acyl carrier protein synthase III. PLOS ONE 10:e0136261. doi: 10.1371/journal.pone.0136261

Marsh, P. (1986). Ptac-85, an E. coli vector for expression of non-fusion proteins. Nucleic Acids Res. 14:3603. doi: 10.1093/nar/14.8.3603

McClean, K. H., Winson, M. K., Fish, L., Taylor, A., Chhabra, S. R., Camara, M., et al. (1997). Quorum sensing and Chromobacterium violaceum: exploitation of violacein production and inhibition for the detection of $\mathrm{N}$-acylhomoserine lactones. Microbiology 143, 3703-3711. doi: 10.1099/00221287-143-123703

Moore, N. M., and Flaws, M. L. (2011). Epidemiology and pathogenesis of Pseudomonas aeruginosa infections. Clin. Lab. Sci. 24, 43-46.

Murugan, E., Kong, R., Sun, H., Rao, F., and Liang, Z. X. (2010). Expression, purification and characterization of the acyl carrier protein phosphodiesterase from Pseudomonas aeruginosa. Protein Expr. Purif. 71, 132-138. doi: 10.1016/j. pep. 2010.01 .007

Ortori, C. A., Atkinson, S., Chhabra, S. R., Camara, M., Williams, P., and Barrett, D. A. (2007). Comprehensive profiling of $\mathrm{N}$-acylhomoserine lactones produced by Yersinia pseudotuberculosis using liquid chromatography coupled to hybrid quadrupole-linear ion trap mass spectrometry. Anal. Bioanal. Chem. 387, 497-511. doi: 10.1007/s00216-006-0710-0

Quadri, L. E., Keating, T. A., Patel, H. M., and Walsh, C. T. (1999). Assembly of the Pseudomonas aeruginosa nonribosomal peptide siderophore pyochelin: in vitro reconstitution of aryl-4, 2-bisthiazoline synthetase activity from PchD, PchE, and PchF. Biochemistry 38, 14941-14954. doi: 10.1021/bi991787c

Raetz, C. R., and Whitfield, C. (2002). Lipopolysaccharide endotoxins. Annu. Rev. Biochem. 71, 635-700. doi: 10.1146/annurev.biochem.71.110601.135414

Ramos-Vega, A. L., Davila-Martinez, Y., Sohlenkamp, C., Contreras-Martinez, S., Encarnacion, S., Geiger, O., et al. (2009). SMb20651 is another acyl carrier protein from Sinorhizobium meliloti. Microbiology 155, 257-267. doi: 10.1099/ mic.0.022079-0

Raychaudhuri, A., Jerga, A., and Tipton, P. A. (2005). Chemical mechanism and substrate specificity of RhlI, an acylhomoserine lactone synthase from Pseudomonas aeruginosa. Biochemistry 44, 2974-2981. doi: 10.1021/bi04 $8005 \mathrm{~m}$
Schafer, A., Tauch, A., Jager, W., Kalinowski, J., Thierbach, G., and Puhler, A. (1994). Small mobilizable multi-purpose cloning vectors derived from the Escherichia coli plasmids pK18 and pK19: selection of defined deletions in the chromosome of Corynebacterium glutamicum. Gene 145, 69-73. doi: 10.1016/ 0378-1119(94)90324-7

Smith, S. (2006). Structural biology. Architectural options for a fatty acid synthase. Science 311, 1251-1252. doi: 10.1126/science.1125411

Spalding, M. D., and Prigge, S. T. (2010). Lipoic acid metabolism in microbial pathogens. Microbiol. Mol. Biol. Rev. 74, 200-228. doi: 10.1128/MMBR. 00008-10

Stead, D. E. (1989). Grouping of Xanthomonas campestris pathovars of cereals and grasses by fatty acid profiling. EPPO Bull. 19, 57-68. doi: 10.1111/j.1365-2338. 1989.tb00129.x

Thomas, J., and Cronan, J. E. (2005). The enigmatic acyl carrier protein phosphodiesterase of Escherichia coli: genetic and enzymological characterization. J. Biol. Chem. 280, 34675-34683. doi: 10.1074/jbc. M505736200

White, S. W., Zheng, J., Zhang, Y. M., and Rock, C. O. (2005). The structural biology of type II fatty acid biosynthesis. Annu. Rev. Biochem. 74, 791-831. doi: 10.1146/annurev.biochem.74.082803.133524

Yuan, Y., Sachdeva, M., Leeds, J. A., and Meredith, T. C. (2012). Fatty acid biosynthesis in Pseudomonas aeruginosa is initiated by the FabY class of $\beta$-ketoacyl acyl carrier protein synthases. J. Bacteriol. 194, 5171-5184. doi: 10.1128/JB.00792-12

Zhao, X., Miller, J. R., and Cronan, J. E. (2005). The reaction of LipB, the octanoyl[acyl carrier protein]:protein N-octanoyltransferase of lipoic acid synthesis, proceeds through an acyl-enzyme intermediate. Biochemistry 44, 16737-16746. doi: $10.1021 /$ bi051865y

Zhu, K., and Rock, C. O. (2008). RhlA converts beta-hydroxyacyl-acyl carrier protein intermediates in fatty acid synthesis to the beta-hydroxydecanoyl-betahydroxydecanoate component of rhamnolipids in Pseudomonas aeruginosa. J. Bacteriol. 190, 3147-3154. doi: 10.1128/JB.00080-08

Zhu, L., Lin, J., Ma, J., Cronan, J. E., and Wang, H. (2010). Triclosan resistance of Pseudomonas aeruginosa PAO1 is due to FabV, a triclosan-resistant enoylacyl carrier protein reductase. Antimicrob. Agents Chemother. 54, 689-698. doi: 10.1128/AAC.01152-09

Conflict of Interest Statement: The authors declare that the research was conducted in the absence of any commercial or financial relationships that could be construed as a potential conflict of interest.

Copyright (C) $2017 \mathrm{Ma}, \mathrm{Wu}$, Cao, Zhang and Wang. This is an open-access article distributed under the terms of the Creative Commons Attribution License (CC BY). The use, distribution or reproduction in other forums is permitted, provided the original author(s) or licensor are credited and that the original publication in this journal is cited, in accordance with accepted academic practice. No use, distribution or reproduction is permitted which does not comply with these terms. 